den basischen Eigenschaften des Sauerstoffs und dessen Vierwertigkeit ergeben. So erinnern dieselben an die vor 57 Jahren publizierte Jugendarbeit B a e y e rs. Damals sprachen seine Versuche für die wechselnde Valenz des Arsens, jetzt dafür, daß der Sauerstoff nicht nur als zweiwertiges, sondern auch als vierwertiges Element funktionieren kann.

Von dem Jahre 1858 an bis zum heutigen Tage hat A d o lf v o n B a e y e r unermüdlich sein ganzes Interesse und seine große Arbeitskraft der Förderung der Wissenschaft zugewandt; er ist in seinen Untersuchungen dem Arbeitsgebiet, welches er von Anfang an gewählt hat, treu geblieben. Auf fast allen Gebieten der organischen Chemie war er bahnbrechend tätig und hat in hervorragender Weise länger als ein halbes Jahrhundert dazu beigetragen, daß sich die organische Chemie so großartig entwickeln konnte, wie dies der Fall war.
Zutreffend dürfen wir deshalb auf sein Schaffen und Forschen G o e th e s schöne Worte anwenden:

Stets geforscht und stets gegründet,

Nie geschlossen, oft geründet,

Altestes bewahrt mit Trene,

Freundlich aufgefaßBt das Neue.

Wenn Adolf von Ba e y e r jetzt auf alles, was er erstrebt und geleistet hat, zurückblickt, so $m u ß$ ihn ein Gefühl hoher innerer Befriedigung und berechtigten Stolzes erfüllen. Wir, seine deutschen Fachgenossen, sind glücklich, daß der große Meister unserem Vaterland angehört, und wir sind alle von dem Wunsch beseelt, daß ihm ein gütiges Geschick noch viele glückliche Jahre bescheren möge, und $\mathrm{ihm}$ auch fernerhin bei voller körperlicher Gesundheit seine bisherige Geistesfrische erhalten bleibe.

\section{Fortschritte in der Chemie der Gärungsgewerbe im Jahre 1914.}

\section{Von A. BaDDREXEL.}

(Gingeg. 29./9. 1916.)

\section{Chemie der Rohstoffe.}

Das Berichtsjahr 1914 wies, wenn auch die letzten 5 Monate desselben nach außen hin unter dem Zeichen des Weltkrieges standen, trotzdem eine sehr fruchtbare wissenschaftliche Tätigkeit auch auf dem weiten Gebiete der Gärung swissen sch aft auf, so daß auch hier ein zusammenfassender Bericht wie in den letzten Jahren die Veröffentlichung der F o r t s c hrit t e dieses Jahres notwendig macht. Hat doch u. a. gerade die Rohstofffrage in der Spiritusfabrikation durch die Kriegsverhältnisse besonderes Interesse erheischt:

Was die Gersten des J a h res 1914 anbelangt, so lauten die diesbezüglichen Berichte über deren Untersuchungen übereinstimmend dahin, daß einerseits der Wassergehalt, wenn zwar im Durchschnitt etwas geringer als im Vorjahre, doch als $\mathrm{n}$ o $\mathrm{r} \mathrm{m}$ a $\mathrm{l}$ anzusprechen ist, andererseits übertrifft der Eiweißgehalt der Gersten denjenigen des Jahres 1913. Die verschiedenen Arbeiten (z. B. von $K$ Wind is $\mathrm{ch}^{1}$ ), H. Wolf $\mathrm{s}^{2}$ ), $\mathrm{H}$ a je k ${ }^{3}$ ), Fries $\mathrm{s}^{4}$ ), J. B. $\mathrm{A}^{5}$ ), Seibrige $\mathrm{r}^{6}$ ), Korits chon e r ${ }^{7}$ ) berichten dementsprechend auch über einen geringeren $\mathbf{E x}$ trakt- und Stärkegehalt, als ihn die 1913 er Gersten aufwiesen. Die Keimversuche ergaben sehr hohe Keimungsenergie bzw. Keimfreudigkeit der Gersten, das Wachstum war im allgemeinen hitzig, was natürlich mit dem hohen Eiweißgehalt der 1914 er Gersten zusammenhängt; über das Aussehen der Gerste wird von $\mathrm{Se} \mathrm{h} \ddot{\text { ö }} \mathrm{n}^{8}$ ) berichtet, daß sie im allgemeinen sattgelb ist und ein auffallend schmales Korn aufweist.

Betreffend Einflu $B$ ünstlicher Trock nung a uf die Stoff-und Zustands verän derungen der Gerste bespricht J. F. Hof $f$ ma $n n^{9}$ ) die Vorteile der Gerstentrocknung, die er als in den meisten Fällen gewinnbringend, in keinem Falle aber schädlich und verlustbringend bezeichnet. Die Trock-

1) Wochenschr. f. Brauerei 31, 403-405 [1914]; Angew. Chem. 28, II, $25[1915]$

2) Bayr. Brauer-Journal 24, 413-414 [1914]; Angew. Chem. 28, II., 25 [1915].

3) Allg. Anzeiger f. Brauerei 30, 1006-1007 [1914]; Angew. Chem. 28, -II, 26 [1915].

4) L. ges. Brauwesen 3r, 445 [1914]; Angew. Chem. 2r, II, 680 [1914].

5). Tagesztg. f. Brauerei 12, 1361 [1914]; Angew. Chem. 28, II, 26 [1915].

6) Wochenschr. f. Brauerei 31, 355 [1914]; Tagesztg. f. Brauerei 12, 1007, 1079 [1914]; Angew. Chem. 27, II, 646 [1914].

7) Brau- u. Malzindustrie 15, 284 [1914]; Angew. Chem. 27, II, $645[1914]$.

8) Wochenschr. f. Brauerei 31, 423-425 [1914]; Angew. Chem. 28, II, 26 [1915].

9) Wochenschr. f. Brauerei 31, 247 u. fl. [1914]; Angew. Chem. 27, II, 547 [1814] nung verh ütedie Verschlechterung ursprünglich feuchter Gerste, verbessere die notreife Gerste, verhindere Verluste durch Atmung und Mikrobenwucherung und damit auch sehr wahrscheinlich eventuelle Extraktverluste. Ein Ungenannter ${ }^{9 a}$ ) berichtet ebenfalls über günstige Erfahrungen in der Praxis mit der Gerstentrocknung auf der Darre bei $35-36^{\circ}$. Co bl it ${ }^{10}$ ) hat praktische Mälzungsversuche mit getrockneter Gerste ausgeführt und schnelle Wasseraufnahme in der Weiche, gleichmäßiges Spitzen und vorzügliches Auflösen bis in die Spitze des Kernes konstatiert. A. CI u B ${ }^{11}$ ) veröffentlicht neuere Erfahrungen über dio Behandlung der Gerste von der Ernte bis zur Verarbeitung unter spezieller Berücksichtigung der Trocknungsfrage. $\mathrm{U} \mathrm{b} \in \mathrm{x}^{\mathbf{1 2}}$ ) berichtet über abnorme Erscheinungen, welche bei der Vergärung von Würze aus bel $22-25^{\circ}$ in der Luft getrockneter Gerste auftreten. Uber die $\mathrm{Sch}$ äd linge der Gersten w u z e l veröffentlicht $\mathrm{H}$. $\mathrm{Z} \mathrm{i} \mathrm{k} \mathrm{e}^{13}$ ) in der Hauptsache eine Zusammenfassung der bisher in der Literatur bekannt gewordenen Erkrankungen, die bei Verarbeitung von Gerste zu Mali auf der Tenne an den Wurzeln des Grünmalzes beobachtet wurden.

Eine systematische mikroskopische Untersuchung über die spezifischen Unterschiede der Gerstenvarietäten Chevallier und Goldthorpe bezüglich ihrer Stärkekörner hat $V$ i n e ${ }^{14}$ ) durchgeführt und gefunden, daß vor allem schon in der Form der Stärkekörner ein Unterschied besteht. Bei der Goldthorpegerste herrscht die $\mathrm{r}$ u n d e bei der Chevalliergerste die e i f ö r $\mathrm{m}$ ig e Gestalt der Stärkekörner vor; noch charakteristischer ist, daß bei der Goldthorpegerste die Zahl der unter 1/10000 Zoll Durchmesser großen Stärkekörner unzweifelhatt größer ist als bei der Vergleichsgerste.

Utber die Zuckerarten des Weizens und der Gerste im Ruhezustande und während der Keimung bringt $\mathrm{A}$. $\mathrm{K} 1 \mathrm{u}$ y $\mathrm{ve}^{15}$ ) (referiert von $\mathrm{B} \mathrm{a} \mathrm{u}$ ) eine umfangreiche Abhandlung, in welcher er für ungekeimte Gerste und Weizen zunächst das Vorhandensein von Raffinose, Rohrzuckerund Monosen (Glucose und Fruchtzucker) feststellen konnte. Ungekeimter Weizen und Gerste enthalten keine Maltose und nur wenig Raffinose; letztere verschwindet zudem während der Keimung vollständig, während die Maltose im Verlaufe der Keimung gebildet wird. Eine $\mathrm{Z}$ u $\mathrm{n}$ a $\mathrm{h}$ m e während der Keimung erleidet der Rohrzucker beim Weizen innerhalb

${ }^{9}$ ) Brau- und Malzindustrie 15, 180-182 [1914].

10) Wochenschr. f. Brauerei 31, 331 u. 332 [1914]; Angew. Chem. 27, II, 645 [1914].

11) Allg. Z. f. Bierbrauerei 42, 253, 265, 277, 295 [1914]; Angew. Chen. 27, II, 546 [1914].

12) Wochenschr. f. Brauerei 31, 319 [1914]; Angew. Chem. 27, II, 645 [1914].

13) Allg. Z. f. Bierbrauerei 42, 469-471 [1914]; Angew. Chem. 28, II, 101 [1915].

14) Journal of the Inst. of Brewing 20, 23 [1914]; ref. in Wochen. schrift f. Brauerei 31, 78 [1914].

15) Biochemische Suikerbepalingen, Leiden 1914; ref. in Wochenschrift f. Brauerei 31, 456 [1914]. 
120 Stunden von 0,73 auf $2,79 \%$ bei der Gerste innerhalb 144 Stunden von 0,76 auf $3,91 \%$; auf der Darre tritt weitere Erhöhung ein; desgleichen $v$ e $r \mathrm{~m}$ e h $\mathrm{r}$ t sich auch der Gehalt an M o n o s e n während des Weichens und Keimens.

F. Schönfeld berichtet auch in Gemeinschaft mit S. Sokolowsk. ${ }^{16}$ ) und mit $G$. S c hönfel e $\mathbf{r}^{17}$ ) über die Mineralbestandteile des Malzes $1913 / 14$ sowie diejenigen von Gerste, Würze, Bier und Hefe, und gibt Anleitungen für die verschiedenen chemischen Bestimmungsverfahren an die Hand. Mit letzterem Verfasser behandelt er auch die Bedeutung der Hefebestandteile für den Lebenszustand der Hefe. Nach einer Arbeit von $H$. L e b e rle und $H$. L ü e $\mathrm{s}^{18}$ ) hat das $M$ äl z e $n$ keinen praktischen EinfluB auf den Gebalt an Ammon ia ks tickst of $f$; dieser schwankt bei verschiedenen Gersten zwischen 0,040 und $0,067 \%$ in der Trockensubstanz. Lagcrung, Düngung oder Beregnung sind ohne Einfluß auf die Menge des Ammoniakstickstoffs.

$\mathrm{Ku} \mathrm{ph} \mathrm{a} \mathrm{19)}$ berichtet über hohen, zum Teil sogar sehr hohen Bitterstoffgehalt des Hopfens (meist bayerischer Herkunft), 16,5 bis 20,7\%. Das Aroma ist mild, rein und edel.

F. $\mathrm{R} \mathrm{a} \mathrm{ba} \mathrm{k}{ }^{20}$ ) hat in einer umfangreichen und eingehenden Arbeit die f $\mathrm{l}$ ü chtigen Bestand teile des Hopfens verschiedener Provenienz und Jahrgänge untersucht, um auf Grund der physikalischen und chemischen Eigenschaften der gewonnenen Hopfenöle Schlüsse auf die Herkunft und das Alter des Hopfens zu ziehen. Es ergab sich, daß das A r o m a des Hopfens durch Ester, die einen großen Teil des Öles ausmachen, hervorgerufen wird. Die flüchtigen Öle des Hopfens bestehen in der Hauptsache aus dem Terpen $\mathrm{M}$ y $\mathrm{r}$ c e $\mathrm{n}$ sowie aus den Estern des $M \hat{y} r c e n a l k o h o l s$ und höberen Fet t 8 ä u r en $\left(\mathrm{C}_{6} \mathrm{H}_{13} \mathrm{COOH}\right.$ bis $\left.\mathrm{C}_{8} \mathrm{H}_{17} \mathrm{COOH}\right)$. Außerdem sind auch noch das Sesquiterpen: $\mathrm{H}$ u $\mathrm{m}$ le $\mathrm{n}$, Spuren von freicr Säure, Formaldehyd sowie allem Anscheine nach auch einige freie Alkohole vorhanden. Sowohl der Estergehalt wie auch der Gehalt an den verschiedenen Ölen schwankt je nach Herkunft und Jahrgang. Doch lassen die Untersuchungen erkennen, daß der Estergehalt der Hopfenöle bestimmter Provenienz oder eines bestimmten Jahres sich sehr ähnlich sind.

Die Ergebnisse der An bau versucheder deutschen Kartoffelkulturstation vom Jabre 1913 wurden wieder von C. v. E c k e b r e c h e r ${ }^{21}$ ) veroffentlicht und zusammengestellt. Der Durchschnitts s tärkegehalt war mit $17,5 \%$ um $0,4 \%$ höher als der des Vorjahres, dagegen um 0,7\% geringer als 1912 . Der höchste $\mathrm{K}$ n ol l e n e r t r a g von $345,1 \mathrm{dz}$ pro $\mathrm{I}$ ha wurde

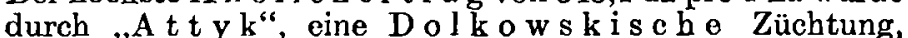
erreicht $(65,5 \mathrm{dz}$ Stärke pro $1 \mathrm{ha})$. Der $S \mathrm{t}$ är k e g e h a l t allor auf 32 Versuchsfeldern verteilter Kartoffelsorten schwankte zwischen 16,0 und $19,8 \%$. Die beobachteten Grenzwerte an Eiweiß waren nach J. F. H of f man und Fr. Pre c k e ${ }^{22}$ ) 1,16 und $3,09 \%$, die Grenzzahlen des Stärkegehaltes haben sich zwar gegen dic des Jahres 1912 gebessert, stehen aber hinter denen der Jahre 1909, 1910 und 1911 zurück.

J. F. H of $\mathrm{mann}$ und C. v. Ecken brecher ${ }^{23}$ ) berichten über Kalidüngungsversuche und stellen fest, daß bei Kalidüngung der Ernteertrag gesteigert werden kann, daß der Stärkeertrag (und der Stärkegehalt) dagegen sich

16) Wochensohr. f. Brauerei 31, 37-38 [1914]; Angew. Chem. 27, U, 213 [1914].

17) Wochenschr. f. Brauerei 31, 245, 493-495 [1914]; Angew. Chem. 27, II, 549 [1914].

18) Z. ges. Brauwesen 37, 321 [1914]; Angew. Chem. 27, II, 547 [1914].

19) Tagesztg. f. Brauerci 12, 1389 [1914]; Angew. Chem. 28, II, 26 [1915].

20) Journal of Agricult. Rescarch Washington II, $\mathrm{Nr}$. 2; ref. in Wochenschr. f. Brauerei 31, 332 [1914]; Angew. Chem. 27, II, 545 [1914].

21) Jahrb. Ver. Spiritusfabr. 14, 61 [1914]; siche auch: Ergänzungsheft d. Z. f. Spiritus-Ind. 37, 1 u. ff. [1914].

22) Ergänzungsheft d. Z. f. Spiritus-Ind. 37, 67-68 [1914].

23) Frgänzungsheft d. Z. f. Spiritus-Ind. 37, 60 [1914]. verringert; in gleicher Weise war auch der Eiweißertrag bei fast allen Versuchen ein kleinerer wenn Kali gedüngt war, als ohne Kalidüngung.

A. B a u d rex e ${ }^{24}$ ) stellt durch gas a n a l y t is c be Un ters u c h u n g e n der bei der Milchsäuregärung entstehenden Gase fest, daß auch bei der $K$ a r t of $\mathrm{fel}$ säuerung durch die Tätigkeit der Milchsäurebakterien kein Verlust an organischer Substanz stattfindet. Nach ihm hat, wie nach früheren Versuchen von $\mathrm{H}$ e $n \mathrm{n}$ e be $\mathbf{r} \mathrm{g}$ zu erwarten war, das Schäumen frisch geriebener Kartoffeln seinen Grund darin, daß durch das Reibseln der Kartoffeln die einzelnen Zellen bloßgelegt werden und so in e r h ö h te m $\mathrm{M}$ a $\mathrm{B}$ e a $\mathrm{t} \mathrm{m}$ en können.

In einer weiteren Arbeit untersucht $\mathrm{B}$ a u d $\mathrm{r}$ e $\mathrm{x}$ e ${ }^{25}$ ) ob bei der Einsäuerung der Kartoffeln gen $\ddot{u}$ gend Dextrose zur Milchsäurebildung vorhanden ist, bzw. ob bei den für die Säuerung in Betracht kommenden Temperaturen genügend Dextrose gebildet wird. Verfasser weist nach, daß sich der Glucosegehalt des Kartoffelsaftes (und damit auch der Kartoffelreibsel) durch die stärkeverzuckernden Enzyme der Kartoffel innerhalb gewisser Grenzen dauernd erhöht, und daß also der jeweilige $Z$ u ckorgehalt der Kartoffel ohne groBe Bedeutung fürdie Kartoffelsäue rung ist. Je höher die Temperatur des Kartoffelsaftes war $\left(31 \mathrm{bzw} .48^{\circ}\right)$, desto größer war die Stärkeverzuckerung. Der frischgewonnene Kartoffelsaft wies die $s \mathrm{t} \ddot{\mathrm{a}} \mathrm{ks}$ te diastatische Kraft auf.

Bei der $Z u$ ckerrübenverarbeitung auf Spiritus, die laut Entscheidung des Bundesrats für das Betriebsjahr 1914/15 zugelassen wurde, waren anfangs verschiedene Schwierigkeiten zu bekämpfon; eine Reihe von Veröffentlichungen bringt diesbezügliche Vorschläge und Ratschläge, die die Verarbeitung der Zuckerrüben behandeln. (G. Fot $h^{26}$ ), P. K $\mathrm{K}$ o $\mathrm{c} z \mathrm{z}^{27}$ ), L üh $\mathrm{d} \in \mathrm{r}^{28}$ ), K. Wind is $\mathrm{c} \mathrm{h}^{29}$ ), C. Förste $\mathrm{r}^{30}$ ), Lang $\mathrm{e}^{31}$ ) u. a.).

N. C. Ort $\nabla$ e d $d^{32}$ ) (referiert von $D$ un $\mathrm{z}$ e) schlägt für Brennereien die Anwendung von $\mathrm{T} \mathrm{a} \mathrm{k} \mathrm{a} \mathrm{-} \mathrm{k} \mathrm{o} \mathrm{j} \mathrm{i} \mathrm{als} \mathrm{Ersatz}$ für die erheblich teueren Malze (aus Gerste, Weizen, Mais usw.) vor. "K o $\mathrm{j} i$ " ist der Name von Reiskörnern, die überwachsen und durchdrungen sind vou dem Mycel des Aspergillus oryzae; "Taka"-koji ist der auf der viel billigeren Weizenkloi e gewachsene Pilz (gesetzl. gesch.) und besagt, daB der Pilz hoc handiastatis cher Kraft sei. Der Verfasser folgert aus seinen Versuchen, daß die Verwendung des Pilzes in der Brennerei an Stelle von Malz, sowohl für Handels- wie für Trinkspiritus (bei mehrmaliger Destillation im Kolonnenapparat) sehr lohnend sei. Die Verzuckerung verlief bei Verwendung von $4 \%$ Koji auf lufttrockenes Maischmaterial bei $45-50^{\circ}$ innerhalb $15-20 \mathrm{Mi}$ nuten, die Gärung ergab eine etwas höhere Ausbeute an Spiritus, als bei Verwendung von Gerstenmalz erzielt wurde. Nähores über das ,Takamineverfa hren" sowic über die ,Aspergillusverzuckerung i m Occiden t" findet sich in $\S 83$ "Handbuch der technischen Mykologie" von Dr. F. L a f a r, Bd. V (1905 bis 1914), S. 331 u. ff., Verlag von Gustav Fischer, Jena (von C. W e h m e r bearbeitet).

Dem Verein der Spiritusfabrikanten in $\mathrm{Deuts}$ c h la $\mathrm{nd}$, Berlin $\left.{ }^{33}\right)$, ist ein Verfahren zur $\mathrm{K}$ on -

24) Z. f. Spiritus-Ind. 37, 109 [1914]; Angew. Chem. 27, II, 467 [1914]

25) Z. f. Spiritus-Ind. 37, 225, 238 [1914]; Angew. Chem. 27, II, 467 [1914].

26) Z. f. Spiritus-Ind. 37, 572, 589, 590 [1914]; Angew. Chem. 28, II, $234[1915]$

27) Z. f. Spiritus-Ind. 37, $572-573$ [1914]; Angew. Chem. 28, II, 262 [1915].

28) Z. f. Spiritus-Ind. 37, 565, 571-572 [1914]; $\Delta$ ngew. Chem. 28, II, 234 [1915].

29) Z. f. Spiritus-Ind. 37, 580-581 [1914]; Angew. Chem. 28, II, 234 [1915].

30) Z. f. Spiritus-Ind. 37, 569 [1914]. [1915].

11) Z. f. Spiritus-Ind. 37, 554 [1914]; Angew. Chem. 28, II, 151

32) Z. f. Spiritus-Ind. 37, 20-21 [1914] (Ưbersetzung); Angew. Chem. 2r, II, 214 [1914].

33) D. R. P. 286 106. Angew. Chem. 28, II, 498 [1915]. 
servierung der Kart of feln patentiert worden, nach welchem den geriebenen einzusäuernden Kartoffeln bei wachstumsgünstiger Temperatur in ben, gegebenenfalls nach Zusatz von zuckerhaltigen Stoffen (z. B. Zuckerrüben oder Zuckerrübenmelasse), eine nach Art des Hefengutes der Brennerei gezüchtete Milchsäurereinkultur zugesetzt wird.

\section{Verarbeitung der Rohstofie.}

(Mälzen und Malz, Maische-und Würzegew in $\mathrm{a} n \mathrm{~g}$.)

W. Romme li4) führte Mälzungsversuche mit Gersten von verschiedenem Gehalt an Eiweiß aus und stellte fest, daß die Gersten mit größerem Eiweißgehait einen höheren $\mathrm{M}$ äl z u n g s c h w u n d als eiweißärmere Gersten haben; die Extraktausbeute war dagegen höher bei letzteren Gerstensorten (es sei verwiesen auf das bereits oben über die Gersten des Jahres 1914 Gesagte).

F. Schönfeld ${ }^{35}$ ) berichtet über die Vermäl z u n g von getrockneter und übersommerter Gerste; die bei getrockneten Gersten gewöhnlich festgestellte Erhöhung der Keimfähigkeit konnte er u. a. nicht feststellen, woraus zu schließen sei, daß die Trocknungswirkung in dieser Hinsicht nicht immer die gleiche ist und nicht stets eine Besserung der Keimungsfreudigkeit hervorbringen muß. Dagegen bewirke die Trocknung stets eine Verhütung der $\mathrm{Ab}$ nahme der Keimfähigkeit, mit der sonst, namentlich bei Gersten mit hohem Feuchtigkeitsgehalt, gerechnet werden muß.

F. E $m s \mathrm{a} n \mathrm{nde} \mathrm{r}^{36}$ ) redet einem einfachen $\mathrm{S}$ y s t e m der Luftwasserweiche das Wort und beschreibt dessen praktische Anwendung in der Brauerei. Nach den bei der schlecht geernteten Gerste des Jahres 1913 gemachten Erfahrungen uber unregelmäßige Keimung behandelt $W$ e in w u $\mathrm{m}^{37}$ ) die Gersten mit und ohne $\mathrm{Kalk}$ was s er; er konnte aber weder in den Laboratoriumsversuchen, noch in der Praxis einen Einflu $B$ auf die Keimfähigkeit feststellen.

Nach dem Verfahren von $\mathrm{O}$. S c h u $\mathrm{z} \mathrm{e}^{38}$ ) wird zum Ansä $\mathrm{u}$ ern von Grünmalz zwecks Beschleunigung der Zersetzungsprozesse, besonders des Eiweißabbaues im Korn, und zur Erzielung einer leichteren Verarbeitung des Malzes zu Bier, ein mit Säurebakterien gesättigter Luftstrom durch das Grünmalz geleitet, um letzteres damit anzureichern.

H. W ill und D. M ed e r e $\mathrm{r}^{39}$ ) bestimmten die $\mathrm{K}$ e i $\mathrm{m}$ $f$ äh ig k e it bei Malzen vom Münchner Typus $(0,75$ bis $4,25 \%$ ), vom Pilsener Typus (von 11-51,5\%). Die Keimfähigkeit ist abhängig von der Temperatur und der Zeitdauer; auch wird sie von der Schimmelbildung im Keimbett mehr oder weniger beeinträchtigt; einfache Waschung mit Sublimatlösung zur Beseitigung der Schimmelpilzbildung war erfolglos.

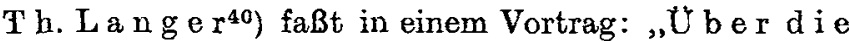
A usbildung von Farbe und Aromabeim Darren des Malzes" seine eigenen und die Ergebnisse anderer Forscher zusammen. Zielbewußte Führung der Tennenarbeit sowie beim Darrprozeß sind hierbei die maßgebenden Faktoren. L a n g e r empfiehlt auf Grund seiner Versuche, den Schwerpunkt der Kontrolle auf das Malz der Oberhorde zu legen, weil dort das Aroma vorbereitet werde; jeder Fehler mache sich dann im fertigen Darrmalz

34) Wochenschr. f. Brauerei 31, 309 [1914]; Angew. Chem. 27, II, 592 [1914].

36) Wochenschr. f. Brauerei 31, 485 [1914]

36) Wochenschr. f. Brauerei 31, 330 [1914]; Angew. Chem. 27, II, 646 [1914]

37) Allg. $Z$. f. Bierbrauerei u. Malzfabrik. 42, 52-54 [1914]; Angew. Chem. 27, II, 291 [1914]

38) O. S chulze, D. R. P. 274 916; Wochenschr. f. Brauerei 31, 356 [1914].

38) Z. ges. Brauwesen 37, 61-64 [1914]; Angew. Chem. 27, II, 291 [1914].

40) Allg. Z. f. Bierbrauerei 42, 33-36 [1914]; Z. ges. Brauwesen 37, 90--93 [1914]; Angew. Chem. 27, II, 291 [1914]. bemerkbar. Anknüpfend an die Mailla r d sche Reaktion wies $L$ intn e $r$ darauf hin, daß in den Farb- und Aromastoffen des gedarrten Malzes den M e la noiden ähnliche Produkte entstünden. Úber diese $\mathrm{M}$ e l a n o i d e berichtet im AnschluB an die Lint nerschen Arbeiten W. $R u c k d$ e s c h e ${ }^{41}$, daß sie beim Erhitzen wässeriger Lösungen von Dextrose und Aminosäuren erhalten werden, ein ähnlicher Prozeß wie beim Darren, wo diese Melanoidine ebenfalls aus Aminosäuren, Polypeptiden und Zucker entstünden.

$J$. F 1 a $\mathrm{m}$ a $\mathrm{n} \mathrm{d}^{42}$ ) behandelt die Frage der $\mathrm{E}$ i w e i $B$ s t of fe des Ma l zes und ihren Einfluß auf die Abläuterung, das Auswaschen der Treber, die Klärung und die Haltbarkeit des Bieres. Die koagulierbaren Eiweißstoffe erscheinen beim Maischen als Oberteig, bei Würzekochen als Bruch. Thre Ausscheidung hängt von verschiedenen Faktoren, so von der Malzbeschaffenheit, von der Wasserzusammensetzung ab.

Die Stärkebesti m $m$ ung i m M a l z auf polarimetrischem Wege wird nach A. C a p p u y u s ${ }^{43}$ nach Zerstörung der Diastase mit Hilfe von $1 / 10^{-n}$. Natronlauge ( $\mathrm{l}^{1 / 2}$ stündiges Stehenlassen) und nachheriger Trennung der neutralisierten Flüssigkeit von der Stärke durch Filtrieren bestimmt; diese Stärke wird sodann in der üblichen Weise verflüssigt, mit etwa $4 \%$ iger Phosphorwolframsäure versetzt, filtriert und polarisiert. Die Methode gibt, wenn es sich um Malze von 75-78\% Extraktgehalt in der Trockensubstanz handelt, gut übereinstimmende und den Tatsachen mehr entsprechende Werte als die Methode von O. S u I . liva n.

O. Schulze ${ }^{44}$ ) wurde ein Verfahren zum Utbertragen von Luftsauerst of f, Bakte. rien, Aromastoffen u. dgl. auf Maischebzw. $W$ ü $\mathrm{r} z$ e geschützt; es ist dad. gekennzeichnet, daß ein Ge. misch von heißen Dampf entwickelndem Wasser mit Luft innerbalb eines geschlossenen Gefäßes über die Oberfläche der kälteren Maische oder Würze geführt wird, derart, daß durch die Kondensation des Dampfes die Luft in feinster Verteilung auf die Flüssigkeit übertragen wird. Durch das Verfahren soll $u$. a. eine gleichmäßige Verteilung von Luft, Bakterien oder Aromastoffen in der Maische oder Würze erzielt werden und zugleich auch durch die verstärkte Sauerstoffzufuhr die Tätigkeit der Enzyme bezüglich des Eiweißabbaues (Peptonisierung, verstärkte Wirkung der Eiweißkraft) angeregt werden.

G. Bode und A. W lo $\mathrm{k} \mathrm{k} \mathrm{a}^{45}$ ) berichten über das Zustandekommen der neuen Vereinbarungen über die Ausführung der Malzanalyse (Bonner Vereinbarungen), die durch die Unterkommission für Malzanalyse der Fachgruppe für Gärungschemie des Vereins Deutscher Chemiker festgestellt worden ist, und teilen deren Wortlaut und analytische Belege in den Erläuterungen ${ }^{46}$ ) mit.

Úber die Bedeutung der Salzodes Bra uwassers und die Notwendigkeit ihrer exakten Bestimmung und Berechnung bringt $W$. W in d is c $\mathrm{h}^{47}$ ) in Fortsetzung seiner früheren umfangreichen Arbeiten praktische Beispiele, woraus er das dringende Bedürfnis ableitet, die Salze des Brauwassers in chemisch wirksame und chemisch unwirksame zu teilen. Zu ersteren gehören vor allem die Carbonate und die übrigen Erdsalze, deren zahlenmäßige Bestimmung im Brauwasser durch eingehende Arbeiten von $K$. W in $d$ is $c h$ und

41) Z. ges. Brauwesen 37, 430-437 [1914]; Angew. Chem. 27, II, 681 [1914].

42) Ann. de la Brass. et de la Distillerie 1913, 529; ref. in Wochenschrift. f. Brauerei 31, 52-53 [1914].

43) Bull. trim. d. l'Assoc. de l'école sup. de Grass. de l'Cniversité de Louvain 1914.

44) D. R. P. 274 777; Angew. Chem. 27, II, 424 [1914]

45) Wochenschr. f. Brauerei 31, 269-272 [1914]; Z. ges. Brau. wesen 37, 372-374, 384-386 [1914]; Angew. Chem. 27, II, 547 [1914].

46) Wochenschr. f. Brauerei 31, 337, 346, 349 [1914]; Angew. Chem. 27, II, 680 [1914].

47) Wochenschr. f. Brauerei 31, 57-60 [1914]; Angew. Chem. 27, II, 292 [1914]. 
seinen Mitarbeitern C. ten Doornkat Koolman ${ }^{48}$ ) und $\mathrm{H}$. $\mathrm{Kr}$ u $\mathrm{m} \mathrm{ha}^{49}$ ). durch Zusammenfassung der einfachen titrimetrischen Bestimmungsmethoden geklärt und durch eine ,n e u e Methode zur Be stimmung der Salze im Wasser" gefördert worden ist.

In betreff der titrimetris chen Wasseran a l y se haben die Verfasser äußerst vereinfachende Methoden vorgeschlagen, die den Gebrauch der bisher meist üblichen, langwierigen Elementaranalyse ersetzen. Die empfohlenen Titriermethoden sind auch auf ibre Genauigkeit entsprechend geprüft worden.

Bezüglich der neuen Bestimmungs- und Berechnungsmethode genannter Verfasser tritt der Hauptunterschied von den alten Methoden namentlich in den Resultaten der kohlensauren und schwefelsauren Magnesia und des kohlensauren Kalks in Erscheinung. Denn während man z. B. früher das Auftreten der schwefelsauren Magnesia gewöhnlich $\mathrm{g}$ a $\mathrm{r}$ n i $\mathrm{ch} \mathrm{t}$ berücksichtigte, hat man auch meist die Menge kohlensaurer Magnesia $\mathrm{z} u \mathrm{~h} \mathrm{o} \mathrm{ch}$, die des kohlensauren Kalks gewöhnlich $\mathrm{z}$ u $\mathrm{n}$ i e d rig befunden. So ergibt sich bei einem Versuchswasser nach der üblichen $\mathrm{Me}$ thode: $\mathrm{MgCO}_{3} 21,1 \mathrm{~g}, \mathrm{CaCO}_{3} 17,5 \mathrm{~g}, \mathrm{CaSO}_{4} 28,7 \mathrm{~g}, \mathrm{MgSO}_{4}$ $0,0 \mathrm{~g}, \mathrm{Na}_{2} \mathrm{SO}_{4} 5,1 \mathrm{~g}, \mathrm{NaCl} 2,6 \mathrm{~g}$, nach der neuen Berechnungs art dagegen: $\mathrm{MgCO}_{3} 7,4 \mathrm{~g}, \mathrm{CaCO}_{3} 32,6 \mathrm{~g}, \mathrm{CaSO}_{4} 8,15 \mathrm{~g}$ $\mathrm{MgSO}_{4} 18,15 \mathrm{~g}, \mathrm{Na}_{2} \mathrm{SO}_{4} 5,1 \mathrm{~g}$ und $\mathrm{NaCl} 2,6 \mathrm{~g}$. Da die schädliche Wirkung der kohlensauren Magnesia gegenüber der Wirkung des koblensauren Kalks belkannt ist, ergibt sich obne weitores, daß das Wasser in diesor Beziehung auf Grund der neuen Berechnung erheblich günstiger zu beurteilen ist, und zwar sowohl in bezug auf seine Wirkung beim Brauen als auch auf seine Verbesserungsfähigkeit beim Entcarbonisieren. Die Verfasser empfehlen neben der "Elementaranalyse" noch die Bestimmung der ,.Kalkkonstanz" des Wassers.

W. Wind i $\mathrm{s} \mathrm{ch}$ und $\mathrm{G} I \mathrm{a} u \mathrm{~b}$ it $\mathrm{z}^{50}$ ) berichten über den $E$ influß der Vordünnunger Maische auf die bei der Untersuchung des Malzes nach dem Kongreßverfahren berechnete Extraktausbeute.

K. Micks ch ${ }^{51}$ ) studierte den Einfluß des Brauwassers auf Hefe, Würze und Bier; er untersuchte $u$. a. Carbonatwässer und carbonatfreie Wässer und stellte die Notwendigkeit einer bestimmten Wasserzusammensetzung des Maischwassers für becondere typische Biere fest.

$\mathrm{Krumha} \mathrm{r}^{52}$ nimmt Stellung gegen die von $\mathrm{R}$. Hermann nach den Arbeiten von $K o u d e l k a s$ im Clu s schen Laboratorium veröffentlichten Ergebnisse auf Grund deren $\mathrm{H}$ or m a n n u. a. die Wasseranalyse nach der Menge der Ionen (in Milligrammäquivalenten) anzugeben empfiehlt. Kru $\mathrm{mhar}$ sieht in dieser Einführung des Ionenbegriffs in das Problem der Wassersalze weder eine erschöpfende Erkiärung, noch oinen Gowinn und tritt den $H$ orm a n schen $H$ y pothosen auch in betreff des Einflusses der Flektrolyte auf die Phosphate entgegen.

Uber den EiweiBgehalt der neuen Würzen berichten F. S c h ö $\mathrm{f}$ eld und $\mathrm{Klos} \mathrm{s}^{53}$ ) und sprechen die Vermutung aus, daß die im allgemeinen eiweißärmeren Würzon infolge der Eiweißarmut höher vergären, falls nicht die Sudhausarbeit entsprechend angepaßt würde. In einer

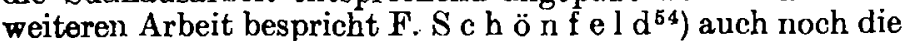
Beeinflussung von $H$ efe und Gärung durch den veränderten Gehalt an Einzelbestandteilen, besonders

48) Wochensohr. f. Brauerei 31, 113-115 [1914]; Angew. Chem. 27, II, 319 [1914].

48) Wochenschr. f. Brauerei 31, 65-67, 73-75, 85--87, 93-96 [1914]; Angew. Chem. 27, II, 319 [1914].

50) Wochenschr. f. Brauerei 31, 1 [1914]; Angow. Chem. 27, IT, 213 [1914].

[1914].

61) Brau- u. Malzind. 15, 71-74; Angew. Chem. 27, II, 320

52) Wochenschr. f. Brauerei 31, 133--136 [1914].

53) Wochenschr. f. Brauerei 31, 96, 137 [1914]; Angew. Chem. 27, II, 320, 388 [1914].

54) Wochenschr. f. Brauerei 31, 145, 197 [1914]; Angew. Chem. 27, II, 389 [1914].
Eiweiß und Extrakt der Würze. Die $M$ i n e r a l bes t and teile der W ürzen haben F. S c hönfeld und S o -

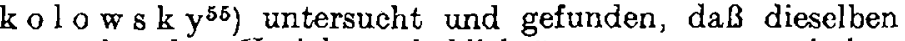
gegenüber dem Vorjahre erheblich ärmer an anorganischen Salzen (Mineralstoffen) sind. Während in den Malzen der Gehalt an Erdalkaliphosphaten löher ais im Vorjabre ist, sind die Würzen dagegen an Kieselsäure und sonstiger anorganischer Phosphorsäure ärmer.

Den Einfluß von manganhaltigem Brauw a s s e $r$ hat $O$. F ü $r \mathrm{n} r$ o $r^{56}$ ) bearbeitet und Vorschläge über die Befreiung desselben vom Mangan gemacht. W i n dis $\mathrm{ch}^{57}$ ) empfiehlt für die Frage, ob $\mathrm{D}$ a m $\mathrm{pf}$ - oder Feu erkochung der Würze stattfinden solle, für erstere Art möglichst große Heizflächen und eine Form der Pfanne, die ein kräftiges Úberstürzen der Würze gewährleistet, dann auch Betätigung von Rührwerken usw.; im übrigen spricht er sich dahin aus, daß die chemischen Unterschiede zwischen Feuer- und Dampfbirnen wohl nicht zu fassen seien. In der Hauptsache sei wohl die Geschmacksfrage ausschlaggebend. Dahingegen redet $\mathrm{Sch}$ h a $\mathrm{z}$ ( o $\mathrm{s}^{58}$ ) aus Nützlichkeitsgründen der Dampfkochung das Wort, während $\mathrm{S}$ c h o ellih or $\mathrm{n}^{59}$ ) mehr zugunsten der feuergekochten Biere spricht.

$\mathrm{G} \mathrm{ribat^{60 } )}$ veröffentlicht seine Erfahrungen über Würzekochen unter Druck; C. Wolf f $\mathbf{f}^{6}$ ) berichtet über die Ei wei Baus s che id ungen (Bruchbildung) in Würzen, die er mit Dampfkochung in breiten Dampfpfannen erhielt.

Di e t. Wieg ma $n n^{82}$ ) empfiehlt für den $H$ opfen kochprozeB zur Vermeidung des Verlustes an Bit terst of $\mathrm{f}$ en durch Kochen und Hopfentreber die Einführung intensiven Rübrens der Würze und die Zugabe der Hauptmenge des Hopfens nicht wie bisher zu Anfang des Abläuterns, sondern zum Schluß.

Genauere Untersuchungen über den Anteil an freien und gebundenen $S$ äuren in Würzen und im Bier haben W. Windisch und $C$. ten Dornkat Koolman angestellt. Wir berichten im vierten Teil darüber (siehe unter Anmerkung 122)).

Nach E. und L. S o b e ${ }^{63}$ ) beträgt der L e c it h in gehalt des Bi eros im Maximum 2,93 g, im Minimum $0,15 \mathrm{~g}$, also im Durchschnitt etwa $1,55 \mathrm{~g}$ im Liter.

Es wurden 26 helle und dunkle, an verschiedenen Orten der Schweiz gebraute Biere auf zwei verschiedene Methoden untersucht, und zwar durch Extraktion der vorher genau neutralisierten Biere mit Ätheralkohol (die Neutralisation erfolgte, um eine event. Lösung der in Äther löslichen anorganischen, sauren Phosphate z. B. prim. Calciumphosphat oder Phosphorsäure selbst, zu verhindern) und durch Fällen der Eiweißstoffe, die die in ihnen enthaltenen Lecithine leicht bestimmen ließen.

R. Seibriger berichtet über die $\mathrm{Malz}-\mathrm{Ger}$ stenextraktdifferenz; er schreibt die Hauptschuld an den in verschiedenen Laboratorien beobachteten Differenzen der wechselnden Art der Untersuchung sowie auch den besonderen Betriebsverhältnissen zu. Für einen und denselben Betrieb seien die Unterschiede zwischen $\mathrm{Mal} \mathrm{z}$ u n d G e r s t e n e x trakt ziemlich konstant, und zwar nur in seltenen Fällen wenig über $1 \%$, gewöhnlich jedoch etwa $0,7 \%$.

(Fortsetzung folgt.)

55) Wochenschr. f. Brauerei 31, 105, 173 [1914]; Angew. Chem. 27, II, 320 [1914].

$[1914]$

6) Z. ges. Brauwesen 37, 369-[1914]; Angew. Chem. 27, II, 548

57) Wochenschr. f. Brauerei 31, 217 [1914]; Angew. Chem. 2r, II, 547 [1914].

58) Wochenschr. f. Brauerei 31, 292 [1914]

59) Wochenschr. f. Brauerei 31, 255 [1914]

60) Wochenschr. f. Brauerei 31, 386 [1914]

61) Wochenschr. f. Brauerei 31, 386 [1914]

62) Allg. Brauer- u. Hopf.-Ztg. 54, 955-957; Angew. Chem. 27, II, 389 [1914]

63) Allg. Brauer- u. Hopf.-Ztg. 54, 1039; Angew. Chem. 27, $\Pi$, 389 [1914].

64) Wochenschr. f. Brauerei 31, 165 [1914]; Angew. Chem. 27, II, $467[1914]$. 
Fortschritte in der Chemie der Gärungsgewerbe im Jahre 1914.

\author{
Von A. BaUdrexei.
}

(Fortsetzung von \$. 440.)

\section{III. (} vorg a n g.

Iu Beiträgen zur Kenntnis der sog. $\mathrm{s} \mathrm{chwarzen}$ Hef en spricht sich $H$. W il $^{65}$ ), gestützt auf Untersuchungen von $\mathrm{F}$. Noldin, gegen die Bezeichnungen: ,Saccharomyces niger, Torula nigra und schwarze Hefe für die beschriebene Pilzart und deren Varietäten aus, da sie in keiner Weise gerechtfertigt seien; vor allem müßte mit den Organismen nach morphologischen Methoden eine höhere Fruktifikationsform erzielt werden.

Einen Vortrag von Lichtenstein-Rosenb $\left(\mathrm{a}^{66}\right)$ referiert $\mathrm{B}$ a $\mathrm{u} d \mathrm{rexe} \mathrm{l}$, wonach eine $\mathrm{D}$ if f e renzierung verschiedener Hefearten mit Hilfe der Agglutinationsmethode ermöglicht wird. Die Blutsera von Kaninchen, denen intravenös Reinkulturen von verschiedenen Hefearten injiziert wurden, lassen nicht nur die verschiedenen Saccharomycesarten (obergärig und untergärig) voneinander unterscheiden, sondern sie gestatten auch, Torulaceen von den Saccharomyceen einwandfrei zu differenzieren.

C. B. C o c h r a n und J. H. P e r k i n $\mathrm{s}^{67}$ ) untersuchten den Einfluß von hohen Temperaturen auf Hefe, und zwar von $35-85^{\circ}$; die Versuchsdauer schwankte zwischen 5 und 30 Minuten. Die Temperaturen über $65^{\circ}$ wirkten im allgemeinen zerstörend auf die lebende Hefe. F. S c h ö n f e l $d^{68}$ ) berichtet über ,Entziehungskuren bei $\mathrm{H}$ ef e $\mathrm{n}^{\text {c: }}$ und gibt verschiedene Wege an, die Hefen an Eiweiß und Mineralstoffen ärmer zu machen (Veränderung der Nährlösung, Lüften der Würze, wärıneres Herführen, große Anstellhefemengen usw.)

L. Buchta ${ }^{69}$ ) hat den Einflu Bdes Lichtes a uf die Sprossung der Hefe studiert und u. a. festgestellt, daß sowohl verschiedene Lichtquellen (Tages licht, elektrisches Licht), wie auch verschiedene Lichtintensitäten die Sprossung von Saccharomyces cerevisiae und Saccharomyces Ludiwigii verschieden $\mathrm{zu}$ beeinflussen verinögen.

Die Sauerstoffwirkung auf die Hefezellvermehrung bearbeitete H. T. Brown ${ }^{70}$ ). Er fand, daß bei beschränkter Sauerstoffzufuhr die Zah der in einem gegebenen Augenblick vorhandenen Hefezellen der Zeit direkt proportional ist. Die Höchstvermehrung der Hefezellen ist, innerhalb gewisser Grenzen der Sauerstoffzufuhr, der Anfangsmenge des Sauerstoffs proportional Die Vermehrungsgeschwindigkeit ist dabei jedoch keine logarithmische, sondern eine lineare Funktion der Zeit. Verfasser hat hierbei auch den Einfluß zugesetzten Alkohols in das Bereich seiner Untersuchungen gezogen und gefunden, daß hierbei eine Zugabe von etwa $8 \%$ Alkohol die Zellenneubildung in negativem Sinne sehr stark beeinträchtigt oder vollkommen aufhebt. Bei geringeren Alkohol mengen wird der Einfluß entsprechend weniger bemerkbar. Aus seinen weiteren Versuchen über die Wärmeerscheinungen bei der Gärung ist zu schließen, daß, wenn wir die Wärmemenge genau ermitteln könnten, die beim Verschwinden ron $1 \mathrm{~g}$ Zucker unter dem Einfluß der Hefe entwickel wird, während sich die Hefezellen in aktiver Vermehrung befinden, diese Wärmemenge annähernd nicht dieselbe sein würde, wie die bei der Vergärung derselben Zuckermengen ohne gleichzeitige Hefevermehrung entwickelte. Der Unter-

65) Z. ges. Brauwesen 37, 13 [1914]; Autoref. nach Zentralbl. $f$. Bakt. II. Abt. 39, I-26 [1914].

66) Wochenschr. f. Brauerei 31, 293 [1914]; Angew. Chem. 27, II, 592 [1914]

67) J. Ind. Eng. Chem. 6, 480 [1914]; Angew. Chem. 28, II, 28 [1915].

68) Wochensohr. f. Brauerei 31, 257 [1914]; Angew. Chem. 27, II, 549 [1914].

69) Zentralbl. Bakt. II. Abt. 41, 340-351; Angew. Chem. 27, II, 549 [1914].

70) Ann. of Botany 28, Nr. 60 [1914]; Übers. in Wochenschr. $f$ Brauerei 31, 272 [1914]. schied würde auf die Energie entfallen, die für die beobachtete Hefevermehrung nötig wäre.

B a u d rexe l $^{71}$ ) bestimmt in Fortsetzung der $\mathrm{L}$ in $\mathrm{d}$ nerschen Arbeiten über die $\mathrm{T}$ a uglichkeit des Athylalkohols als Nährstoff für ver. s c h i e d e n e Pil z e die genauen Mengen Alkohol, die die drei Organismen: Saccharomyces farinosus, Oidium lactis und Kahmhefe B Henneberg, innerhalb bestimnter Zeiten als Nahrung aufzunehmen imstande sind, und berechnet genau die Kohlenstoffmengen, die die Pilze aus dem Alkohol pro Tag als Nahrung zu entnehmen vermögen.

C. B. C o c h r a n und J. H. P e r k i n s ${ }^{72}$ ) stellten beim Studium des Einflusses geringer Äthylal koholmengen a uf di e $\mathrm{G}$ ä $\mathbf{r}$ u $\mathrm{g}$ von Rohrzuckersirup (D. 1,2112-1,26) bei $20^{\circ}$ fest, daß ein Gehalt von 1 oder weniger Volumprozent an Äthylalkohol die Gärung der Zuckerlösungen in bemerkenswerter Weise beschleunigt. Erst stärkere Alkoholkonzentrationen (über 1,25\% Alkohol) verzögern die Gärung steigend mit dem Alkoholgehalt.

Uber den Einflu $\mathrm{O}$ organischer Säuren a uf die Hefe berichten W. Buromsky sorvie H. Z j ckes. B urom sk $\mathrm{y}^{73}$ ) stellt fest, dal die Hefe in Nährlösungen mit organischen Säuren keine Zymase, dagegen mehr oxydierende Enzyme bildet; sie kann sich allmählich an den sauren-Nährboden gewöhnen, gewinnt dann aber erst allmählich, event, nach einer Reihe von Generationen, wieder, mit Zucker zusammengebracht, die Fähigkeit, Zucker zu vergären. $\mathrm{Z} \mathrm{i} \mathrm{c} \mathrm{k} \mathrm{e}{ }^{74}$ ) bespricht den Einfluß folgender Säuren auf Hefen und Bakterien: Ameisen-, Essíg-, Milch-, Butter-, Wein-, Bornstein-, Oxal-, Apfel- und Zitronensäure.

Th. Bokorn $\mathrm{y}^{35}$ ) hat den $\mathrm{E}$ in $\mathrm{fla} \beta$ der schon von anderer Seite erwähnten $M$ angansalze a uf $d$ i e H ef e dahin erklärt, daß er die Uns ch äd li chk eit darauf zurückgeführt, daß die Hefe diese Salze nicht aufnimmt, während sie z. B. Eisen-, Nickel- und Kobaltsalze zu resorbieren vermag. In einer anderen Arbeit ${ }^{76}$ ) behandelt derselbe Verfasser die $\mathrm{Fäh}$ igkeit d er $\mathrm{H}$ ef e, $\mathrm{Me}$ tallsalze, namentlich $\mathrm{Sch}$ ermetallsalze $\mathrm{zu}$ binden.

F. S c h ö $\mathrm{nfeld}$ und $\mathrm{G}$. S c h ö n f e l d e r ${ }^{77}$ ) vertreten in einer Arbeit: ,Di e Minera $\mathrm{Ib}$ estand teile der Hefe und ihre Bedeutulng für den Lebens zustand derselben", den Standpunkt, daß die Bruchhefe mehr mit Eiweiß und Mineralbestandteilen gesättigt sei als die Staubhefe, und daß besonders charakterisierte Hefen mit ausgeprägten Eigenschaften auch bei Utberführung in einen neuen Betrieb ihre Eigenheiten möglichst beizubehalten streben. Der in verschiedenen Hefen (Brauerei-Reinhefen) gefundene niedrige G l y k o g e n g e h a lt wird von F. S c hönfeld und E. K ün z e $1^{78}$ ) darauf zurückgeführt, daß im letzten .Jahre, bei Verwendung der eiweißreichen Malze, die Hefe im allgemeinen Neigung zur stärkeren Bruchbildung und damit zu geringerer Aufspeicherung von Glykogen gezeigt hat.

Unter den Umsetzungsprod ukten bei der Tätigkeit der Hefe wird von Fern bach und S c ho e $n^{29}$ ) Brenztra u bens ä u re festgestellt.

$V$ o is in e t ${ }^{80}$ ) konnte den im bitteren Wein aufgefundenen $\mathrm{Bac}$ illus a maracylus in fast allen Wäs-

71) Wochenschr. f. Brauerei 31, 400 [1914]; Angew. Chem. 28, II, 28 [1915]. [1915].

) J. Ind. Eng. Chem. 6, 141 [1914]; Angew. Chem. 28, II, 28

73) Zentralbl. f. Bakt. II. Abt., 42, 530-557 [1914]; Angew. Chem. 28, II, 150 [1915].

74) Allg. Z. f. Bierbrauerei 43, 1-4; Angew. Chem. 28, II, 151 [1915].

75) Chem.-Zttg. 38, 1290 [1914]; Angew. Chem. 28, II, 151 [1915].

76) Allg. Brauer- u. Hopf.-Ztg. 54, 170-175, 1155-1158; Angew. Chen. 27, II, 470 [1914].

77) Wochenschr. f. Brauerei 31, 245 [1914]; Angew. Chem. 27. II, 549 [1914].

78) Wochenschr. f. Brauerei 31, 9 [1914]; Angew. Chem. 27. IJ. 215 [1915].

70) Ann. de Brasserie 1913, 554.

80) Compt. rend. 158, 195 [1914]. 
sern feststellen. Er vergärt Zucker und verschiedene mehratomige Alkohole, namentlich Glycerin und Mannit; als Produkte entstehen Wasserstoff, Äthylalkohol, Acrolein, $\mathrm{CO}_{2}$, Ameisen-, Fssig-, Milch- und Bernsteinsäure.

Die cellulosevergärenden Bakterien sind nach $\mathrm{K} \mathbf{r}$ on li k ${ }^{81}$ ) in aerobe und anaerobe, thermophile Bakterien zu unterscheiden; nach Prings hei m wurden neben Wasserstoff und Kohlensäure bei der anaeroben Gärung ausschließlich Essigsäure und Ameisensäure gebildet, was vielleicht zu einer neuen technischen Fabrikation dieser Säuren führen wird.

Der B a c ill us Delbrü $\mathrm{c} k$ i wird nach P. Hen ne. b e r g ${ }^{82}$, entgegen anderweitigen Angaben, durch $\mathrm{t}^{4}$ berschreitung seiner höchsten Säuerungstemporaturen von $50^{\circ}$ mehr oder weniger geschädigt, so daß zwar bei dieser Temperatur die Maischen (Fabrik- und Laboratoriumsversuche) an der Oberfläche noch gesäubert werden, im Innern aber nur schwache Säurebildung auftritt; bei längerer hoher Säuerungstemperatur stirbt der Bacillus ab. Dasselbe Ergebnis zeigte sich auch boi einem angeblich thermophilen Milchsäurepilz. Nat ürliche Reinzucht-Milch $\mathrm{s} \ddot{\mathrm{u}} \mathrm{r} e \mathrm{bakterien}$ zeigen allerdings bisweilen anch noch bei höheren Temperaturen noch Säuerungsvermögen

P. Not $\mathrm{t} \mathrm{in}^{83}$ ) verfolgt die schon von $\mathrm{I}_{4}$ ind et und A m man studierten Einwirkungen von $Q$ u e c ksil. ber a uf di e alkobolische Gärung und erklärt die gewonnenen Resultate dahin, daß der Einfluß des Quecksilbers im wesentlichen mechanischer Art ist. Wenn die Menge der Quecksilbersalze, deren Bildung man nicht verhindern könne, nicht zu groß sei, so wirke das Quecksilber als fester Körper, der die Cbersättigung mit Kohlensäure verhindere infolgedessen könue die Hefe aber besse $r$ a $t m e n$ und som it a u ch besser wa chsen; auf der anderen Seite schienen sowohl die Quecksilbersalze wie auch das Metall selbst weder auf die Gärfunktion, noch auf das Auswahlvermögen der Hefe einen Einfluß zu haben. Diese Frklärung durch clie rein mechanische Wirkung des Quecksilbers fand eine Stütze in der Beobachtung, daß der Verfasser u. a. fand, daß die Hefenernte mit der Größe der Berührungsfläche der Gärflüssigkeit mit dem Quecksilber stieg. Die Tatsachen selbst waren im wesentlichen schon früher von $\mathrm{Dum}$ as, $\mathrm{Schulz}, \mathrm{Bernacki}, \mathrm{Mann}$ u n d P o k o r n y festgestellt worden.

Nach C. Ri che t $^{84}$ ) gewöhnen sich Milchs äu re bakterie $n$ in wenigen Tagen so sehr an giftige Salze (K-Arseniat, K-Phosphat, K-Sulfat, K-Selenat, K-Bromat u. a.), daß das Wachstum sich gegenüber normalen Lösungen sogar steigert. Eine Vererbung derart, daß die Bak terien an Giftstoffe gewöhnt worden können, konnte nicht festgestellt werden.

In dem $\mathrm{Brots} \mathrm{ch}$ i m elpilz Rhizopus nigricans haben R. A. Gortner und A. F. Blakeslee $e^{85}$ das Vorkommen eines $G$ if $t \mathrm{~s}$ t of $\mathrm{fes}(4 \%)$ festgestellt der bei intravenöser Einführung in Kaninchen deren $\mathrm{T}$ o d unter allen Symptomen der Anaphylaxis verursachte. Die letale Dosis für Kaninchen ist I Teil auf 225000 Teile Körpergewicht.

Ưber neuere Arbeiten, die sich mit den F n z y me n von Mikroorganis men beschäftigt haben, sei zunächst eine Arbeit von C. S. $\mathrm{H}$ u d s o n und A. S. P a i $n e^{86}$ ) referiert, durch welche festgestellt wurde, daß die I $n$ v e $r$ $t$ a s e a s $\mathrm{H}$ e f e Sucrose vollständig zu Invertzucker hydrolysiert; diese Reaktion ist aber nicht umkehrbar. Des weiteron veröffentlicht $C$. $S$. $H$ u d $s$ o ${ }^{87}$ ) eine verbesserte Methodezur Herstellung starker Invertasolösungen aus ober- und untergäriger Hefe;

81) Zentralbl. f. Bakt. u. Paras. 28, 20. 1914].

83) Compt. rond. 15\%, 1005; ref. in Wochenschr. f. Brauerei 31, 55 [1914].

84) Compt. rend. 158, 764

85) Vers. Am. Chem. Soc., Rochester, Sept. 1913; Angew. Chem. 27, II, 215 [1914]

86) J. Am. Chem. Soc. 36, 1571-1581 [1914]; Angew. Chem. 28, IT. $106[1915]$.

87) .r. Am. Chem. Soc. 36, 1566; Angew. Chem. 28, II, 106 [1915]. während dic Invertase aus ersterer Hefe Raffinose in Melibiose und Lävulose spaltet, wird dieselbe Zuckerart durch die Unterhefe-Invertase in Dextrose, Lävulose und Galaktose zerlegt. Dic Bereitung der Invertaselösungen geschieht in folgender Weise: $10 \mathrm{~kg}$ Hefe werden mit 101 Teitungswasser und $500 \mathrm{ccm}$ Toluol durchgearbeitet und dann bei gewöhnlicher Temperatur 5 Tage der Autolyse überlassen; nach Bleizuckerzusatz (bis kein Niederschlag mehr entsteht) wird das Filtrat mit $\mathrm{H}_{2} \mathrm{~S}$ vom Blei befreit. Die sauren Filtrate werden dann sofort (24 Stunden genügen meistens) durch einen Kollodiumsack dialysiert. Dîe ctialysierten Lösungen werden entweder mit Toluol haltbar gemacht (nach $1 / 2$ Jahr zeigen sie noch die Hälfte der enzymatischen Kraft) oder im Vakuum unter $30^{\circ}$ zur Trockne verdampft.

Nach $T h .13$ o k o r $\mathrm{n}^{88}$ ) geht die mit Hilfe der proteolytischen Enzyme vonstatten gehende Hefeverdauung nie über eine gewisse niedere Grenze hinaus. Es müssen wohl zwei versehiedene proteolytische En z y m e angenommen werden, die beide in saurer Lösung, aber bei verschiedenem Säuerungsgrad wirken. Das tierische Pepsin ist dem proteolytischen Enzym der Hefe weit überlegen.

Nach C. Ne u berg und Czapski89) besitzt auch obergärige Hefe $\mathrm{Ca} \mathrm{rb}$ ox y las $\mathrm{e}$; wie aus einer weiteren Arbeit von C. N e u berg und $N$. I w a $n$ of $f^{80}$ ) erhellt, unterliegt die scheinbar widerstandsfähigere $\mathrm{C}$ a r bo $\mathbf{x}$. l a s e, deren Widerstandskraft bekanntlich auch verschiedenen physikalischẹn Finflüssen gegenüber der Zymase größer ist, nicht in dem Maße wie Zymase der Wirkung gewisser Antiseptica, organischer und anorganischer Natur z. B. Natriumfluorid, Quecksilberchlorid, Formaldehyd, Phenol, Thymol. Gärkräfftiger Hefemacerationssaft, mit solchen antiseptischen Stoffen versetzt, vergor noch Brenztraubensäure, während Rohrzucker und Dextrose nicht mehr zersetzt wurden.

Durch Tonerdehydrat, das nach W. H Welke r und J. M a r s h a l $^{91}$ ) durch Fällen einer verdünnten Ammoniakalaunlösung mit Ammoniak als Gallerte erhalten wird, können fast alle Enzyme aus wässerigen Lösungen vollständig ausgefällt werden.

Die Invertasebi I d ng des Bacillus Delbrücki kann nach $H$. E u le r und $H$. Cra me ${ }^{92}$ ) durch Vorbehandlung des Bacillus mit zuckerhaltigen Nährlösungen stark gesteigert werden. Während die Enzymbildung in Dextroselösung unter normalem Wachstum vor sich geht, vermehren sich die Zellen in Lactoselösungen bei gleicher Enzymbildung nur schr schwach.

S. S. Graves und P. A. Kob e ${ }^{93}$ ) empfehlen als Ersatz für Toluol beim Arbeiten mit Enzymen Trikresol, und zwar bei gewöhnlicher Inkubationsdauer für ' $\mathrm{r}$ y p s i n, Pepsin, Urease und Dia. stase 0,5\%igen, für Erepsin und Invertase 0,2\%igen Zusatz: Ricinussamenlipase wird durch 0,2\%ige Iösung in ihrer Wirkung a uf Athylbutyrat und Triacetin verlangsamt; Pankreatin erleidet dagegen scheinbar eine gewisse Boschleunigung der Zerlegung von Äthylbutyrat.

$\mathrm{Buch}$ ner und $\mathrm{M}$ e is e nhei mer haben die Eintstehung des Glycerins aus Traubenzucker im Hefensaft gefunden, was neuerdings durch $M$. O p p e $n$ h e i m er ${ }^{01}$ der die Glycerinbildung als eine von der Gärkraft der Hefe bzw. des Hefensaftes a b hängige Nebenreaktion bei der alkoholis chen Gärung crklärt, bestätigt wurde. Als besonders gutes $\mathrm{A}$ u s g a $\mathrm{ng}$.s $\mathrm{m}$ a t e$r$ i a l vermögen Dioxyaceton und Glycerinaldehyd Glycerin zu bilden. Unter den flüchtigen Bason der bei der Hefonautolyse entstehenden Nebenprodukte des $\mathrm{Z}$ erfalls der Aminos äuren werden nach N. Iwa-

88) Allg. Brauer- u. Hopf.-Ztg. 54, 2533-2534; Angew. Chem. 28, II, 105 [1915].

89) Biochem. Zeitschr. 6\%, 9 [1914].

90) Biochem. Zeitschr. 67, 1 [1914]

01) J. Am. Chem. Soc. 35, 822; Angew. Chem. 2r, II, 84 [1914].

92) Biochem. Zentralbl. 58, 217

93) J. Anı. Chem. Soc. 36, 751--758; Angew. Chem. 28, II, 28 [1915].

y4) Z. physiol. Chem. 89, 63 [1913]. 
n of $\mathrm{f}^{95}$ ) Amine, wahrscheinlich Amylamin und Trimethylamin, gebildet.

Nach neueren Untersuchungen $S$. K o s t y $t \mathrm{sch}$ e $w^{\text {r s }}{ }^{96}$ ) entsteht durch lebende oder getötete Hefe aus Acetaldehyd außer nach der $\mathrm{C}$ an $\mathrm{n}$ i z $\mathrm{z}$ a $\mathrm{r}$ o schen Reaktion wahrscheinlich noch durch Wasserstoffanlagerung Äthylalkohol bzw. Athylalkohol und Essigsäure. Aus weiteren Untersuchungen von $K$ os t y $\mathrm{t} \mathrm{ch}$ e $w$ und $W$. B r ill a n t ${ }^{97}$ ): ,Uber die stickstoffhaltigen Stoffe im Macerationssaft der Hefe," können nach erfolgter Hydrolyse der Eiweißstoffe durch starke Autolyse des Preßeaftes unter gewissen Bedingungen wieder synthetische Vorgänge eintreten, die sich namentlich auf den nach der $S t$ u $t$ z e $r$ schen Methode mit Kupferhydroxyd fällbaren Stickstoff (Proteinstickstoff) beziehen.

$\mathrm{H}$ ans $\mathrm{Eul}$ e $\mathrm{r}^{98}$ ) berichtet über die Rolle des Glykogens bei der Gärung clurch lebende Hefe. E. S a I . $\mathrm{k}$ ow s k y hat, durch die in dieser Arbeit angewandte Glykogenbestimmung nach Schönfeld und K r a m p f veranlaßt, diese Methode nachgeprüft und sie für d u r c h a u s u n z I äss i g befunden, da, auch wenn man das Hefegummi beseitigt, das Glykogen dureh Auflösung eines Teils der Zellmembran einen unberechenbaren Gewichtszuwachs einer sich wie Glykogenverhaltenden Substanz erfährt.

Bei einer Untersuchung: „t'ber die gleichzeitige Veranderung des Gehaltes an Glykogen, an Stickstoff und an Enzymen in der lebenden Hefe" hat sich nach $\mathrm{Sixten} \mathrm{K}$ ull be $\mathrm{r} \mathrm{g}^{99}$ ) ergeben, daß im allgemeinen die für verschiedene Hefen von $S \mathrm{ch}$ önfeld bestät ig t e $\mathrm{R}$ e $\mathrm{g} \in \mathrm{l}$ auch für eine und dieselbe Reinkulturhefe besteht, nämlich: hoher Glykogengehalt beringt niederen Stickstoffgehalt. Weiterhin zeigte sich, daß bei vorbehandelter untergäriger Hefe durch die Gärung nicht eine Verminderung, sondern eine starke Erhöhung cies Glykogengehalts eintritt. $\mathrm{K}$ u $11 \mathrm{~b}$ e $\mathrm{g}$ hat hierbei aber obige Einwände Salkowskys gegen das Schönfeld $\mathrm{Kr}$ a m $\mathrm{p}$ f sche Glykogenbestimmungsverfahren nicht berücksichtigt.

Läßt man $\mathrm{H}$ e f e a af $\mathrm{F}$ u r f u ro l einwirhen, so bilcet sich nach C. J. L i n t n ex and C. J. v. Li e big (II. Mitteilung ${ }^{100}$ ) neben Furfurolalkohol Furvltrimethylenglykol, $\mathrm{C}_{4} \mathrm{H}_{3} \mathrm{O} \cdot \mathrm{CHOH} \cdot \mathrm{CH}_{2} \cdot \mathrm{CH}_{2} \mathrm{OH}$. Die Versuchsansteller nehmen hierzu an daß zwischen dem Furfurol uud dem intermediär bei der Gärung entstehenden Acetaldehyd eine Aldolkondensation stattfindet, und dieses letztere Aldol sodam zu dem Glykol reduziert wird:

$\mathrm{C}_{4} \mathrm{H}_{3} \mathrm{OCHO}+\mathrm{CH}_{3} \mathrm{CHO}+\mathrm{H}_{2} \rightarrow \mathrm{C}_{4} \mathrm{H}_{3} \mathrm{O} \cdot \mathrm{CHOH} \cdot \mathrm{CH}_{2} \cdot \mathrm{CH}_{3} \mathrm{OH}$.

$\mathrm{O}$. $\mathrm{M} \circ \mathrm{h} \mathrm{r}^{\mathbf{1 0 1}}$ ) unterwirft die mikrocalorimetrischen Versuche $R \mathbf{u} b$ n e $x$ s und seine sich daran knüpfenden Berechnungen einer eingehenden kritischen Besprechung und knüpft daran eigene ma $\mathrm{kr}$ oc a $\mathrm{l}$ o ri me $\mathrm{tr}$ is c h e Un tersuchungen über die Wärmeentwicklung bei der Hefengärung und bei enzymatischen Vorgängen. Nach dem Verfasser wird die Wärmeentwicklung bei der Rohrzuckervergärung durch die einsetzende Gärwärme etwas, wenn auch nur wenig herabgesetzt, und zwar infolge eines endothermen Vorgangs: der Inversion des Rohrzuckers. Die Versuchslösungen waren folgende: Traubenzucker, Invertzucker, Rohrzucker und Würzen (helle Würze, Porterbier und Weißbierwürze) Bezüglich des Einflusses der Hefenmenge auf den Verlauf der Gärung hat Verfasser gefunden, daß die hierbei entwickelte Wärmemenge z. B. bei 10 oder $20 \mathrm{~g}$ Hefe dem Gewicht der verwanden Hefe $n$ icht proportional ist. Als Gärwärme für (l g) Maltose hat $\mathrm{M} \circ \mathrm{hr}$ durch Messungen der Gärungskohlensäure 134 Grammcalorien gefunden.

๑5) Biochem. Zeitschr. 63, 359 [1914].

96) Z. physiol. Chem. 89, 367, 402 [1913].

97) Z. physiol. Chem. 91, 372 [1913].

98) Z. physiol. Chem. 92, 75 [1914].

s9) Z. physiol. Chem. 88, 109 [1912].

100) Z. physiol. Chem. 92, $340-360$ [1914].

101) Wochenschr. f. Brauerei 31, 394-399, 412-417 [1914]; Angew. Chem. 28, II, 27 [1915].
Úber die Verwendung ron Nachgärungshefen bei der Herstellung von Porter und ihre Erfolge in der Praxis wurde durch W. Ro m m e lin) berichtet; er hat nachdem schoi C l a u s s en festgestellt hat, daß der dem Porter eigentümliche Geruch und Geschmack durch gewisse, die weitgehencle Vergärung der Biere hervorrufende Torulaceen (Brettanomyces) verursacht werde, diese Nachgärungshefen (sekundäre Hefen) des englischen Porter gezüch tet und als Porternachgärungshefen sowohl deutschen und österreich-ungarischen wie auch ausländischen Bravereien mit bestem Erfolg zur Verfügung gestellt. Die B e r l in e r Versuchs-und Lehrailstalt für Brauerei bringt ciiese Porternachgärungshefen zum Verkauf.

Ein Verfahren zur Umzüchtung und Ver. mehrung y on Bi erh ef e wurde ner Versuchs- und Lehranstalt für Brauerei, Berlin ${ }^{103}$ ) patentiert; es ist dadurch gekennzeichnet, daß Bierhefe unter Hinzufügung einer Aussaat von Kahmhefe unter Durchliuften zur Ver. mehrung gebracht wird.

Durch ein Patent der $\mathrm{R}$ a d i o te ch $\mathrm{n}$ is chen $\mathrm{St} u$ diengesellsehaft m. b. H. Charlotten b u r ${ }^{104}$ - Berlin wird die übliche Durchlüftung bei der L ufthefefabrikat ion dadurch ersetzt, daß statt des Einblasens von Luft durch die Würze $\mathrm{r}$ a d i o a $k$ tive $S u b$ s t a n z e n zur Verwendung gelangen.

Nach seinem Patent der Becco-Gesell s ch a $t$ f ür $\mathrm{H}$ ef everedelung $\mathrm{m}$. b. H. ${ }^{105}$ ) wird Preßhefe oder Bierhefe dadurch veredelt, daß man sie aus einer Maische aus Rohrzucker und verkleistertem Roggen - und Kartoffelmehl zur Umgärung bringt.

Wahl-Henius, Inatitute of Fermentogie, Chi. cago ${ }^{106}$ ), haben sich ein Verfahren patentieren lassen zur Herstellung von haltbarem, sjch nicht tru übenden Bi er, insbesondere von Flaschenbier das Verfahren ist dadurch gekennzeichnet, daß der Würze oder dem Bier während seiner Bereitung ein $1-2 \%$ Säure, insbesondere Milchsäure, enthaltender Peptaseauszug aus Malz oder dgl. zwecks Abbaus und Löslichmachung der Eiweißstoffe zugesetzt wird.

Nach A. R. Ling und A. B. Woold ridg $\mathrm{e}^{107}$ ) soll der Brauproze $\beta$ dadurch verbessert werden, da $\beta$ man die in üblicher Weise hergestellte Würze mit dem gesamten nötigen Hopfen in einer geschlossenen Kupferpfanne mit Dampfbeheizung bei etwa $0,4-0,45$ Atmosphären $1 / 4$ Stuncie lang kocht; hernach wird ciie Würze noch im Vakuum etwas konzentriert

Um Hefe von einer allerdings nur geringen In $\mathrm{fe} \mathrm{k}$ ti on $v$ on $B$ a $k \mathrm{t}$ erien $n$ zu reinigen, schlagen J. $\mathrm{Cr}$ o lbois und C. Ukmar $r^{108}$ ) vor, dieselbe mit Ozon zu behandeln; Hefe sei gegen Ozon widerstandsfähigker als Bakterien.

Eine Reihe von Abhandlungen befaßt sich mit der Klärung und Reinigung von $A$ bw $\ddot{a}$ ssern a us $G \ddot{a}$. rungsbetrieben, die gewöhnlich Kolloide gelöst enthalten; es sei verwiesen $u$. a. auf die verschiedenen Veröffentlichungen von $R \circ h l$ a $d^{109}$, von $\left.G u t h^{110}\right) u$. a.

$P$ a u l Dreverhoff ${ }^{111}$ ), Grimma i. Sa., hat sich ein Verfabren, das in erster Linie für die Malzbereitung bestimmt ist, patentieren lassen: $W$ e i chverfahren für Getreide, Sämereien und Pilze unter $\mathrm{Zufuhr}$ yon $\mathrm{N}$ ah $\mathrm{rsal}$ zen, dadurch gekennzeichnet, claß'clem Weichwasser nacheinander Alkalien und Säu-

102) Wochenschr. f. Brauerei 31, 88 [1914]; Angew. Chem. 27, II, 294 [1914].

103) R. D. P. 269426.

104) D. R. P. 275432.

105) D. R. P. 277639

106) D. R. P. 275 213; Angew. Chem. 2r, II, 469 [1914]

107) J. of Inst. of Brewing 81 [1914].

108) Ann. de l. Brass. 17, 13 [1914]

109) Z. ges. Brauwesen 37, 413 [1914]; Angew. Chem. 27, II, 64I

[1914]; Papierfabrikant 12, 361 [1914]; Angew. Chem. 27, II, 517 [1914]; Kolloid-Ztg. 14, 195 [1914]; Angew. Chem. 27, II, 512 [1914]; Seifensiederztg. 41, 310 [1914]; Angew. Chem. 2\%, II, 512 [1914] Z. f. Spiritus-Ind. 3\%, 58 [1914]; Angew. Chem. 2r, II, 387 [1914] 110) Angew. Chem. 27, II, 253, 612 [1914].

111) D. R. P. 271 938; Angew. Chem. 27, II, 139 [1914]. 
ren zur Bildung von Nährsalzen innerhalb des Getreides usw. zugeführt werden. Dr. $R$ e in h old $\mathrm{K}$ us s e r o w ${ }^{112}$ ), Sachsenhausen, untermischt nach seinem Mälzereiverf a h r e n das keimende Getreide mit auflockernden, indifferenten Stoffen z. B. Torffaser, Sägespänen, Haferschalen u. dgl., um ein leichteres Entweichen der Keimungsgase zu befördern

Die Versuchs - und Lehranstalt für Braurei , Berlin ${ }^{113}$ ) hat verschiedene Patente für Ausführungs formen des durch das Patent 245607 geschützten Ve r fahrens zur Herstellung von Yoghurt. bi e r erhalten. Ein Verfahren zur Veredelung des Bieres an Geschmack und Vollmundig-

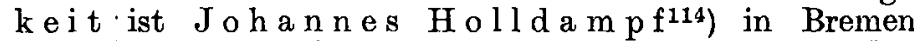
patentiert; das Verfahren ist dadurch gekennzeichnet, daß die Hefekultur in den Lagerfässern ausgeführt und vollendet wird, wobei die Hefe in einer Vorkultur in vollprozentiger Stammwürze bis zur Reife gebracht und dann dem Bier im Lagerfa $\beta$ zugesetzt wird. Hierbei kann die Vorkultur auch zunächst in ungehopfter Würze durchgeführt werden.

Nach dem Brauverfahren mit enthülstem Malz von G. Fr. H e r $m$ a $n n^{115}$ ), Elberfeld, wird enthülstes Grobschrot auf einer einfachen Unterlage von Gewebestoff (Nesseltuch, Gaze oder dgl.) als Auslöse- und Filtermaterial zugleich verwendet, wobei die durch die Unterlage hindurch filtrierte Würze nur Zellengewebe der reinen Schrotkerne hindurchläßt, ohne daß dabei Hülsen oder Hülsenextrakt in die Würze gelangen. Die Würze soll dadurch weder Trübung, noch auch den Hülsenbeigeschmack aufweisen.

\section{Gärungserze ug n is se.}

F. E m s länd e $\mathrm{r}^{116}$ ) empfiehlt zur $\mathrm{S}$ ä u re bes t i m$\mathrm{mung}$ i $\mathrm{m} \mathrm{Bi}$ e $\mathrm{r}$ und bei dessen Bereitung die elektrometrische Methode der Messung der Was serstoff i o nenkonzentration; nach dieser Methode wird der Fehler, der beim Farbenumschlag durch den Indicator bedingt ist, ausgeschaltet, weil hier die Neutralität auf einer physikalischen Grundlage, nämlich auf der Dissoziationskonstante des Wassers beruht.

Auch $H$. L e berle und $H$. L ü e $\mathrm{rs}^{117}$ ) führten mit dieser elektrometrischen Methode erfolgreich Säurebestimmungen durch. In ,Versuchen über die Anwendung von Leitfähigkeitsmessungen bei der Untersuchung von Bier" faßt H. L ü e $\mathrm{r} \mathrm{s}^{118}$ ) die bisherigen Ergebnisse dieser Methode zusammen und empfiehlt die elektrische Methode besonders für praktische Betriebe, um feine Unterschiede, bedingt durch Veränderungen im Brauwasser, Beschaffenheit des Malzes, Vergärung usw. systematisch zu ergründen. Von Interesse ist die Feststellung, daß sich nährstofffreie und nährstoffhaltige Zuckerlösungen bei der Säurebildung durch die Hefe nicht unterscheiden.

Die mutmaßlichen und tatsächlichen Reaktionen, die die dem Bier zugesetzten löslichen $\mathrm{E}$ is e n ver bi nd u n g e n durchmachen, sind verschiedener Art. F. Sch önf el $d^{119}$ ) nimmt an, daß außer den direkt zugegebenen Eisensalzen noch solche vorhanden sind, die erst sekundärer Art, ja auch tertiärer Art sind. Auch die Gerbsäure der Biere bleibt nicht ohne Einfluß; es scheiden sich grüne oder blaue feine Flocken von gerbsaurem Eisen ab, die einen

112) D. R. P. 280 131; Angew. Chem. 27, II, 547 [1914].

113) D. R. P. 271 833; Angew. Chem. 27, II, 72 [1914]; D. R. P. 271 834; Angew. Chem. 27, II, 72 [1914]; D. R. P. 271 835; Angew. Chem. 27, II, 72 [1914]; D. R. P. 276486.

114) D. R. P. 278941 .

115) D. R. P. 278482 ; Angew. Chem. 27, II, 646 [1914].

$116) Z$. ges. Brauwesen 3y, 2, 16, 27, 164 [1914]; Kolloid-Z. 13, 156-169 [1913] u. Kolloid-Z. 14, 44 [1914]; Angew. Chem. 27, II, $141,293[1914]$.

[1914].

117) Z. ges. Brauwesen 31, 177 [1914]; Angew. Chem. 27, II, 389

118) Z. ges. Brauwesen 31, 79, 210,227 [1914]; Angew. Chem. 27, II, 290,390 [1914].

119) Wochenschr. f. Brauerei 31, 321 [1914]; Angew. Chem. 27, II, 647 [1914]. unangenehmen Geschmack haben. Schließlich aber konnte Schönf eld einen direkten Phosphorsäureverlust des Bieres, wahrscheinlich durch Eisen-Phosphorsäureverbindunger hervorgerufen, feststellen. Bezüglich des geeignetsten Eisensalzes, das sich auch noch nach etwa 6 Wochen zum größten Teil im Bier gelöst vorfand, erwies sich das Ferrum oxydatum saccharatum dem Ferrum albumosum bzw. peptonatum überlegen. Die Versuche sind mit schwach vergorenen und schwach gehopften Bieren durchgeführt worden. Man bedient sich am zweckmäßigsten des alkalisch reagierenden Eisenzuckers zu dunklen Süßbieren. Das preußische Finanzministerium hat in der norddeutschen Brausteuergemeinschaft diesen Zusatz gestattet.

Der Kolloidgehalt in Bieren ist nach Robe $r$ t $\mathrm{M} \mathrm{a} \mathrm{rc}^{120}$ ) im Mittel mit etwa $7,2 \%$ der Refraktion des ausgeschüttelten Bieres gefunden worden. Die hellen Biere sind im allgemeinen kolloidärmer als die dunklen; M a r c kommt zu dem Schluß, daß der K o l I o i d g o h a l t keinen EinfluB auf den Geschmack der $B$ i e r e hat, was auch R. E m s l a n d e r gutheißt. Außerdem hat Verfasser auch die Beobachtung gemacht, daß der Refraktionswert eines Bieres, das (durch dreitägiges Stehen an der Luft) schal wird, bedeutend abnimmt. M a res Feststellungen beweisen aufs neue, daß die in jedem normal bereiteten Bier enthaltenen kolloiden Eiweißkörper einer allmählich fortschreitenden Koagulation unterliegen, so daß also auch ein normal bereitetes, a us gereiftes Bier stets noch - bezüglich der kolloidalen Eiweißkörper gewissen langsamen Veränderungen unterworfen ist.

Die schon früher von $W$. W i n $d$ i s $c h$ gemachte Beobachtung, daß sich die Hefe im gegebenen Milieu eine ihr günstigste Säurequantität $\mathrm{zu}$ verschaffen weiß, wird durch W. Windis ch und C. ten Doornkat Kool ma ${ }^{121}$ ) bestätigt. Die Verfasser konnten ihre Säurebestimmungen nicht ohne weiteres nach den in der Weinanalyse üblichen Methoden durchführen, da es sich ja beim Bier bzw. der Würze um zum Teil erheblich kleinere Säuremengen handelt als beim Wein. Sie extrahierten die Untersuchungsflüssigkeiten (Würzen und Bier) mittels des umgeänderten Perforators nach $P$ a $r$ th eil-R os e und konnten hiermit freie wie gebundene $S$ äuren bestimmen, was für ciie Aufklärung der Säurebildungsverhältnisse während der Gärung von großer Wichtigkeit ist; wenn auch die Würze nur sehr geringe, nicht genau festzustellende Mengen an frei e r $S$ ä u re enthält, während sie an gebundener organischer Säure beträchtlichere Quantitäten aufweist, so konnte doch durch den Vergleich der Gesamtsäuremengen in Würze und im halbfertigen und fertigen Bier festgestellt werden, daß während der Würzegärung ebenso wie während der Weingärung eine Neubildung an organischen Säuren stattfindet. Der Säuregehalt des Bieres hängt ab: 1. von dem verwendeten Malz, 2. vom Salzgehalt des Brauwassers, 3. vom Maischverfahren, 4. von der Heferasse, 5 . von der Behandlungsweise des halbfertigen Produktes während der Iagerung. Von den flüchtigen Säuren findet sich der Hauptanteil unter den freien, von den fixen Säuren der Hauptanteil unter den gebundenen Säuren. (SchluB folgt.)

\section{Berichtigung}

zum Aufsatz von Borrmann, Neuere Arbeitsmethoden zum Waschenund Destillieren von Flüssigkeiten (S. 377):

S. 379, rechts, Zeile $20: \ldots$ bei $r$. . fällt fort.

S. 379, rechts, Zeile 26: statt: Siedepunkt des Benzols ... lies: Siedepunkt des Anilins ..

S. 384, links oben: Fig. 12 ist um $180^{\circ}$ zu drehen.

120) Kolloid-Z. 14, 181 [1914]; Angew. Chem, 27, II, 470 [1914].

121) Wochenschr. f. Brauerei 31, 225, 235, 252, 275, 295, 303, 311 [1914]; Angew. Chem. 27, II, 592 [1914]. 
säure, Zum Versilbern von Aluminium schlägt P. F. L. Béve n ot ${ }^{36}$ ) vor, einen dünnen galvanischen Niederschlag einer Nickel-Silberlegierung herzustellen, auf den dann erst ein dicker Silberüberzug erfolgen soll. Das Verfahren von $\mathrm{C}$ a $\mathrm{n}$ a $\mathrm{c}$ und $\mathrm{T}$ as $\mathrm{s}$ il y zum Abbeizen von Aluminium ist bereits in einem früheren Bericht erwähnt. Wie aus den Angaben der deutschen Patentschrift ${ }^{37}$ ) ersichtlich, soll der zu vernickelnde Gegenstand erst in lauwarmem Wasser gespült und dann in ein 2\%iges Cyankaliumbad gebracht werden. Hat er hierin eine mattsilberweiße Farbe erreicht, so kommt er in ein Bad, das aus $500 \mathrm{ccm}$ destilliertem Wasser und $500 \mathrm{~g}$ Salzsäure, in der I g Eisen gelöst wurde, besteht. Nach Auftreten eines silberweißen Metallglanzes auf dem Aluminium wird dieses wieder gespült und ins Nickelbad übergeführt. Letzteres enthält pro Liter Bad $50 \mathrm{~g}$ Nickelchlorid und $20 \mathrm{~g}$ Borsäure. Die Spannung soll 2,5 Volt, die Stromdichte 1 Ampere betragen. N. J. C a n a c und $\mathrm{E}$ T a s s il $y^{38}$ ) heben hervor, daß der mattgraue Nickelüberzug anf derartig vorbehandeltem Aluminium sehr gut haftet; was durch die netzartige Anätzung der Oberfläche durch das Eisenchlorid erklärt wird. Zur Ausnutzung des Verfahrens hat sich bereits eine Gesellschaft ${ }^{39}$ ) gegründet. Bei Nachprüfung der Vorschrift fanden W o $\mathrm{g}$ i n $\mathrm{z}$ und $\mathrm{H}$ a $I 1 \mathrm{a}^{40}$ ), daß die starksaure Eisenlösung das Aluminium intensiv angreift. Die Eisenzwischenschicht haftet zwar sehr gut, aber die Vernickelung wird rauh und uneben. ZurErzeugung glatter Nickelüberzüge mußte stark vernickelt und mit Zugscheiben nachbehandelt werden. Die Versuche zur Beseitigung dieser Nachteile führten zu keinem befriedigenden Ergebnis. Sie empfehlen an Stelle des Canac-Tassilyverfahren folgende Methode. Die entsprechend vorbehandelten Aluminiumwaren werden zunächst $1-2$ Minuten lang in ein $30-40^{\circ}$ warmes Bad aus $40 \mathrm{~g}$ Kaliumzinkcyanid, $10 \mathrm{~g}$ Cyankalium, $150 \mathrm{~g}$ Soda und $50 \mathrm{~g}$ Ätznatron pro Liter Wasser verbracht, in welchem sie bei Verwendung von Eisenanoden einen gleichmäßigen grauen Zinküberzug erhalten. Dadurch sind dieselben für die Weiterbehandlung in einem beliebigen Bad geeignet. Wünscht man ganz solide Galvanisierungen, bei denen auch bei längerer feuchter Lagerung ein Durchschlagen von Ausblühungen aus dem Aluminium nicht zu befürchten ist, so muß die Zinkschicht erst im cyankalischen Bad verkupfert und dieser Überzug im saueren Kupferbad verstärkt werden, wobei man für feinkörnige Niederschläge dem Plastikbad zweckmäßig etwas Glycerin zusetzt.

\section{Bäder und Niederschläge.}

Zur Bestimmung der freien Blausäure sowie des ungebundenen Cyankaliums in galvanischen Bädern empfiehlt G. E. F. L u n d e $1^{1^{4}}$ ) eine schnelle und genaue Methode, die auf der 'Titration der ammoniakalisch gemachten Lösung mit Nickelammonsulfat bei Gegenwart von Dimethylglyoxim beruht. Die scharlachrote Nickelglyoximverbindung fällt erst aus, wenn das Cyan als Nickeldoppelsalz gebunden ist. Die genaue Arbeitsweise geht aus einer gemeinsam mit J. A. B $\mathrm{r}$ i d $\mathrm{g} \mathrm{m} \mathrm{a}^{42}$ ) gemachten Veröffentlichung hervor. Danach soll die Nickellösung 15,3 g Nickelammoniumsulfat sowie $2 \mathrm{ccm}$ konz. Schwefelsäure pro Liter enthalten. $50 \mathrm{ccm}$ dieser lösung entsprechen $1 \mathrm{~g}$ Cyankalium. Von dem Dimethylglyoxim löst man $8,9 \mathrm{~g}$ in $1195 \%$ igen Alkohols und verwendet für jede Titration hiervon $0,5 \mathrm{ccm}$. Die Reaktion wird außer von Kaliumzinkcyanid von keinem der in galvanischen Bädern üblichen Doppelcyaniden gestört.

Von P. M a r i no ist früher bereits der Vorschlag gemacht worden, den Bädern Magnesiumborocitrat zuzusetzen, worüber nähere Angaben aus der inzwischen bekannt gewordenen deutschen Patentschrift ${ }^{43}$ ) zu ersehen sind. Neuerdings empfieblt derselbe Autor ${ }^{44}$ ), die abzuscheidenden $\mathrm{Me}$ -

36) Franz. Pat. 466193.

37) D. R. P. 276257, Kl. 48a.

38) Compt. rend. 158, 119 [1914]; Bll. Soc. chim. 15, 595 [1914].

39) Chem.-Ztg. 38, 232 [1914]

40) Metall 1914, 375 .

41) Metallurg. Chem. Eng. 12, 340 [1914].

42) J. Ind. Eng. Chem 6, 554 [1914]; Angew. Chem. 28, II, 158 [1915].

43) D. R. P. 276258 , Kl. 48a.

44) Engl. Pat. 28953 von 1912 talle in Form ihrer Salicylate zu verwenden. Der Elektrolyt soll daneben etwa die gleiche Menge salicylsaures Ammonium oder Alkali enthalten und mit genügend Ammoniak bis zur deutlichen alkalischen Reaktion versetzt werden. Vor der Zugabe des Ammoniaks fügt man dem Bad etwas Sulfosalicylsäure zu. Für die Abscheidung verschiedener Metalle und Legierungen wird die Zusammensetzung der Bäder angegeben.

Einen alkalisalzfreien Elektrolyten benutzt G. S a c e r d o t $\mathrm{e}^{45}$ ). Außer dem Salz, dessen Metall abgeschieden werden soll, enthält das Bad noch Eisen- und Mangansulfat, sowie Aluminiumacetat.

Blei. Auf der Suche nach Zusatzstoffen, welche die Abscheidung oines feinkrystallinischen, weichen Niederschlags aus Bleibädern begünstigen sollen, hat $F$. C. M a t h e $\mathrm{r} \mathrm{s}^{46}$ ) weitere Körperklassen $\mathrm{zu}$ seinen Versuchen herangezogen und ist dabei über Stoffe wie Tannin, Casein, verschiedene ätherische Öle und Fette allmählich bei Produkten angelangt, von denen bisher wohl noch kein Galvaniseur Gebrauch gemacht hat. Die Resultate waren auch bei den mit Fleischextrakt, Auskochungen von Vogelfedern und exotischen Pflanzen wenig zufriedenstellend. Als gut arbeitend wird ein Bad beschrieben, das pro Liter Wasser $100 \mathrm{~g}$ Bleiacetat, $40 \mathrm{~g}$ Ammoniumperchlorat und $25-50 \mathrm{ccm}$ Eisessig enthält. In letzterem wird der Extrakt von $12,5 \mathrm{~g}$ Aloe gelöst. Man elektrolysiert, ohne zu filtrieren, mit einer Stromdichte von 0,4 Ampere und setzt während der $\mathrm{Ab}$ scheidung noch etwas Essigsäure mit der Aloeabkochung zu. An Stelle des essigsauren Elektrolyten können nach F. C. Mathers und B. W. Co ckru m${ }^{47}$ ) auch milchund ameisensaure Bäder verwendet werden. Das Ammonperchlorat läßt sich außerdem durch sulfonaphthalinsaures Natrium ersetzen. Der Niederschlag aus solchen Bädern wird durch die großen Mengen von Additionsstoffen leicht brüchig. Von der Abscheidung des Bleis aus salpetersauren Lösungen, die Gallussäure als einen sowohl anodisch wie kathodisch wirkenden Depolarisator enthalten, hat $R$. G a r t e $\mathrm{n}$ e is $\mathrm{te}^{48}$ ) bei einer elektroanalytischen Bestimmungsmethode Gebrauch gemacht.

Cadmium. Eine Ưbersicht über die bisher für galvanische Cadmiumniederschläge angegebenen Verfahren geben F. C. $\mathrm{M}$ a th e $\mathrm{r}$ s.und $\mathrm{H}$. M. M a $\mathbf{r}$ b l $\mathrm{e}^{49}$ ); sie berichten über ihre eigenen auf die Gewinnung möglichst glatter Ưberzüge abzielenden Versuche. Geprüft wurden fluorwasserstoffsaure, kiesel- und borfluorwasserstoffsaure, sowie überchlorsaure Bäder mit jeweils 4\% Cadmiumgehalt. Von den Zusatzstoffen erwiesen sich Pepton, Leim, Nelkenöl am besten geeignet, also die gleichen Körper, deren günstiger Einfluß bereits bei den Bleibädern beobachtet wurde. (Schluß folgt.)

\section{Fortschritte in der Chemie der Gärungsgewerbe im Jahre 1914.}

Von A. Baudrexel.

(SChinB von S. 448.)

F. Schönfeld und R. Klos s122) untersuchten zahlenmäßig die $S$ t i ckst of fent $n$ a $h$ meaus Wür zen von obergärigen Bieren durch die Hefen und bestätigten zunächst, daß die Hefen, genau so wie bei den Kohlenhydraten, den Stickstoff der Würzen nicht restlos assimilieren, sondern denselben je nach Art der Hefen bzw. der Würzen entuehmen. So fanden sie, daß von dem überhaupt assimilierbaren Anteil an Stickstoff

bei Berliner Weißbier. . . . . . . . . . $=2 / 3=66 \%$ bei Doppelbier............. . . . . $=1 / 2=50 \%$ bei Caramelbier . . . . . . . . . . . . $=1 / 3=33 \%$ bei untergärigem, hellem Bier . . . . . . $=1 / 3=33 \%$ bei untergärigem, dunklem Bier weniger als $=1 / 3=30 \%$ assimiliert werden.

45) Amer. Pat. 1109181.

46) Metallurg. Chem. Eng. 12, 714 [1914]; Trans. Am. Electrochem Soc. 24, 315-329.

47) Metallurg. Chem. Eng. 12, 714 [1914].

48) Chem.-Ztg. 37, 1281 [1913]

49) Metallurg. Chem. Eng. 12, 714 [1914]

122) Wochenschr. f. Braverei 31, 345-346 [1914]; Angew. Chem. 27, II, 646 [1914]. 
Uber den Lecithingehalt des Bieres wurde schon oben eine Arbeit von E. und $\mathrm{L}$. S o bel kurz besprochen (siehe Anmerk. $\left.{ }^{63}\right)$ ).

Nach Adalb. Podlog a $\mathrm{r}^{123}$ ) bietet ein durch ein Koordinatensystem dargestelltes $\mathrm{D}$ i a $\mathrm{g} r$ a $\mathrm{mm}$ eine wesentliche Vereinfachung der Bestimm ung von Al kohol und Extrakt im Bier mittels des Zeissschen Eintauchrefraktometers. Die Fehlergrenze des Diagramms, das die entsprechenden Werte nach der Refraktometerbestimmung direkt abzulesen gestattet, ist gegenüber der gewichtsanalytischen Bestimmung für Alkohol 0,005--0,015 und für Extrakt 0,002-0,008 gegenüber Danzers $s^{\top}$ Tabellen (umgerechnet auf N.-Temp. 17,5 für Alkohol 0,01-0,02 für Extrakt 0,005-0,015.

Uber die Grundlagon der Alkoholdestil l a t i o $n$ berichtet $E$. C h en a $r^{124}$ ); weiterhin veröffentlicht derselbe Verfasser eine $T h$ eorieder fraktio. nierten Destillation der Gemische von Alkohol und Wasse ${ }^{125}$ ).

E. L ü h $d$ e $r^{126}$ ) hat eine Anzahl exakt durchgeführter Brennereiausbeutebestimmungen in ges chlos enen $\mathrm{G} a ̈ \mathrm{r} b$ o t $\mathrm{t}$ i c h e $\mathrm{n}$ mit Kohlensäurewäsche durchgeführt und für je $100 \mathrm{~kg}$ eingemaischte Stärke 63,4 bis $66,6 \mathrm{l}$ (im Mittel 66,5 l) reinen $A$ lkohols erhalten; es entspricht dies einer Ausbeute rund $93 \%$ der Theorie.

Theo R. Ha as mann, Den $\mathrm{H}$ a a $\mathrm{g}^{127}$ ) berichtet über koloniale alkoholische Gärungserze.u g n is se; E. D untze ${ }^{128}$ ) hat im Anschluß an Vorversuche, die in bezug auf die $\mathrm{Herstellung}$ von $\mathrm{Wh}$ is ky nach amerikanischem Verfahren günstige Resultate zeitigten, Einlagerungen von Branntwein im Großen vorgenommen. Er wählte hierzu etwa $200 \mathrm{l}$ fassende Fässer aus amerikanischem und slavonischem Eichenholz, die innen angekohlt sind. Zur Befüllung derselben dienten verschiedene Sorten Kartoffelsprit, sog. "Alkohol", und fuselreicher Kartoffelrohspiritus (ca. 50 volumprozentig).

Der Inhalt der Fässer soll regelmäßig nach bestimmter Zeit auf seine Veränderung in geschmacklicher wie chemischer Beziehung (Alkohol-, Extrakt-, Ester-, Aldehyd-, Furfurol-, Säure-, Fuselölgehalt) untersucht werden.

E. $D u n t \mathrm{ze}^{129}$ ) berichtet über Trinkbranntwein in unseren afrikanischen Kolonien und teilt die Untersuchungsergebnisse von mehreren, zum Teil aus dem Ausland eingeführten Branntweinen (namentlich Whiskysorten) mit.

E. P. H äussler ${ }^{130}$ ) veröffentlicht Beiträge zur Kenntnis des „Reifens" von Kognak und untersuchte die Veränderungen, die Weindestillate erleiden, wenn sie in Glasstöpselflaschen mit Eichenholzstücken längere Zeit zusammengebracht werden. Außer der Veränderung des Gohaltes an Alkohol, höheren Alkoholen, Estern, gesamter und flüchtiger Säuren, Extrakt, Asche und reduzierenden Stoffen konnte er im Weindestillat auch Vanillin - aus dem Eichenholz stammend - nachweisen. Estor, flüchtige und Gesamtsäure, reduzierende Stoffe und Extrakt zeigten eine $\mathrm{Z}$ u $\mathrm{n}$ a $\mathrm{h}$ m e; während der Gehalt an höheren Alkoholen gleichblieb, wies der Äthylalkoholgehalt eine

123) Allg. Z. f. Bierbrauerei 42, 408, 410 [1914]; Angew. Chem. 28, II, 27 [1915].

124) Bll. Soc. chim. [4] 15, 592 [1914]; Angew. Chem. 28, II, 106 [1915].

[1915].

Bll. Soc. chim. [4] 15, 844 [1914]; Angew. Chem. 28, II, 106

126) Z. f. Spiritus-Ind. 37, 275 [1914]; Angew. Chem. 27, II, 647 [1914].

127) Z. f. Spiritus-Ind. 37, 361, 374 [1914]; Angew. Chem. 27, II, 585 [1914].

128) Jahrb. Ver. Spiritusfabr. 1914, 153.

129) Korrespondenz der Abt. f. Trinkbranntwein u. Likörfabrikation Jhrg. V, Nr. 2 [1914]. [1914]. gewisse $A$ b n a $h$ me auf, die Vf. im wesentlichen auf $O x y$. dation durch Luftsauerstoff und Absorption durch das Eichenholz zurückführt.

Carl Gilg131), Berlin-Lichterfelde, ist ein Verfahren zur Herstellung alkoholarmer, kohlens äurereicher Getränke patentiertworden, nach welchem die zu vergärenden, zuckerhaltigen Flüssigkeiten mit Nektarien führenden Blüten und zugleich mitf $e$ ing e mah len em Lein kuch enm e h l versetzt werden. Die in den Nektarien von Blüten vorkommenden Hefen werden durch die im Leinkuchenmehl vorkommenden Organismen in für die Gärung zweckdienlicher Weise unterstützt, und zugleich gelangen durch die Leinkuchen noch andere geschmackgebende und schaumbildende Stoffe in die Getränke.

Wie Versuche von $G$. W üstenfeld und $\mathrm{Ch}$. F o e h $\mathrm{r}^{132}$ ) ergeben haben, können durch Ausnutzung der Winterkälte (A u s r i e r e $\mathrm{i}$ m Freien) um so höher konzentrierte Alkoholessige gewonnen werden, je tiefere Temperaturen angewandt werden. Bei $10^{\circ}$ werden Älchen durch Ausfrieren getötet.

$R$. Heinzelma $n^{133}$ ) bringt eine literarische $\mathbf{Z u}$ sammenstellung der Erfindungen auf dem Gebieteder Essigfabrikation.

A. $M \circ h r^{134}$ ) beschreibt ein neues $V$ e r f h $\mathrm{r} \theta \mathrm{n} \mathrm{zur}$ Kondensation bei der Schnellessigfabri. $\mathrm{k}$ a $\mathrm{t}$ i o $\mathrm{n}$, bei welchem durch vollständige Ausnutzung der Alkohol- und Essigdämpfe eine Mehrausbente von etwa $12 \%$ Essig gewährleistet wird.

Die A. Marktscheffelsche Kondensat i o n s a n la ge in Osnabrück wird von $G$. Wüsten f el $\mathrm{d}^{135}$ ) untersucht und nach dem Prüfungsergebnis als mustergültig und rentabel bezeichnet.

Uber die Herstellung des Malzessigs durch ein verbessertes Orleansverfahren berichtet J. T. $S, t$ e i d e $\left.1^{136}\right)_{2}$ (übersetzt von $H$. R o s s m a $n$ ).

Als Ersatz für den Sa uerteig137) zum Brotbacken wird die Untermischung von $0,1 \%$ Es s i g unter den mit gekochten, gepellten Frischkartoffeln verkneieten Brotteig empfohlen.

$H$. Wü st en f e l d ${ }^{138}$ ) beschreibt Methoden zur Wi e derbelebung außer Betrieb gesetzter Es $\mathrm{sig}$ b ild ner. Zur Entfaltung des verborgenen Iebens der Essigpilze empfiehlt er namentlich : frische, leichtassimilierbare Nährstoffe, Alkohol in Form von Maische, Wärme und Luft sowie geeigneten chemischen und physikalischen Zustand des ruhenden Bildners.

Eine wesentliche Beschleunigung der Essigsäurebildung soll nach G. Bertrand und R. S a z er a c ${ }^{139}$ ) durch Gegenwart ron $\mathrm{M}$ angansalzen erreicht werden.

Utber ein einfaches photographisches Verfahren im Dienste der biologischen An a l y s e berichtet $P$. Lind $n$ e ${ }^{140}$ ). Es lassen sich hierdurch sehr schnell und einfach mit Hilfe eines parallelen Lichtbündels (von einer Bogenlampe) direkte Schatten und Halbschattenbilder von Plattenkulturen, K le ingärversuch en usw. sowie auch von lebende $n \mathrm{Org}$ a $\mathrm{n}$ i s $\mathrm{m} \in \mathrm{n}$, die in schmalen Glasküvetten vor das (Gaslicht-) Papier gebracht werden, herstellen. [A. 113.]

131) D. R. P. 273034.

132) Essig-Ind. 18, 51 [1914].

133) Essig-Ind. 18, 199 u. ff. [1914].

134) Essig-Ind. 18, 173-176; D. R. P. 270320 [1914]; Angew. Chem. 27, II, 215 [1914].

135) Essig-Ind. 18, 149-151; D. R. P. 252150 [1914].

136) Pure Products I, 10; ref. in Essig-Ind. 18, 432-428 [1914].

137) Essig-Ind. 18, 456 [1914].

188) Essig-Ind. 18, $476[1914]$.

[1914].

. Soc. chim. [4] 15, 627 [1914]; Angew. Chem. 27, II, 716

140) Z. f. Spiritus-Lnd. 37. 301, 307 [1914]; Angew. Chem. 27, II, 647 [1914]. 